\title{
Pharmacogenetic testing for adverse drug reaction prevention: systematic review of economic evaluations and the appraisal of quality matters for clinical practice and implementation
}

Saowalak Turongkaravee ${ }^{1}$ (D) Jiraphun Jittikoon², Onwipa Rochanathimoke ${ }^{1}$, Kathleen Boyd ${ }^{3}$, Olivia Wu ${ }^{3}$ and Usa Chaikledkaew ${ }^{4,5^{*}}$ (1)

\begin{abstract}
Background: Genetic testing has potential roles in identifying whether an individual would have risk of adverse drug reactions (ADRs) from a particular medicine. Robust cost-effectiveness results on genetic testing would be useful for clinical practice and policy decision-making on allocating resources effectively. This study aimed to update a systematic review on economic evaluations of pharmacogenetic testing to prevent ADRs and critically appraise the quality of reporting and sources of evidence for model input parameters.

Methods: We searched studies through Medline via PubMed, Scopus and CRD's NHS Economic Evaluation up to October 2019. Studies investigating polymorphism-based pharmacogenetic testing, which guided drug therapies to prevent ADRs, using economic evaluation methods were included. Two reviewers independently performed data extraction and assessed the quality of reporting using the Consolidated Health Economic Evaluation Reporting Standards (CHEERS) guidelines and the quality of data sources using the hierarchy of evidence developed by Cooper et al.

Results: Fifty-nine economic evaluations of pharmacogenetic testing to avoid drug-induced ADRs were found between 2002 and 2018. Cost-utility and cost-effectiveness analyses were the most common methods of economic evaluation of pharmacogenetic testing. Most studies complied with the CHEERS checklist, except for single studybased economic evaluations which did not report uncertainty analysis (78\%). There was a lack of high-quality evidence not only for estimating the clinical effectiveness of pharmacogenetic testing, but also baseline clinical data. About $14 \%$ of the studies obtained clinical effectiveness data of testing from a meta-analysis of case-control studies with direct comparison, which was not listed in the hierarchy of evidence used.
\end{abstract}

\footnotetext{
* Correspondence: usa.chi@mahidol.ac.th

${ }^{4}$ Social and Administrative Pharmacy Division, Department of Pharmacy,

Faculty of Pharmacy, Mahidol University, 447 Sri-Ayuthaya Rd, Payathai, Ratchathewi, Bangkok 10400, Thailand

${ }^{5}$ Mahidol University Health Technology Assessment (MUHTA) Graduate

Program, Mahidol University, Bangkok, Thailand

Full list of author information is available at the end of the article
}

(c) The Author(s). 2021 Open Access This article is licensed under a Creative Commons Attribution 4.0 International License, which permits use, sharing, adaptation, distribution and reproduction in any medium or format, as long as you give appropriate credit to the original author(s) and the source, provide a link to the Creative Commons licence, and indicate if changes were made. The images or other third party material in this article are included in the article's Creative Commons licence, unless indicated otherwise in a credit line to the material. If material is not included in the article's Creative Commons licence and your intended use is not permitted by statutory regulation or exceeds the permitted use, you will need to obtain permission directly from the copyright holder. To view a copy of this licence, visit http://creativecommons.org/licenses/by/4.0/. The Creative Commons Public Domain Dedication waiver (http://creativecommons.org/publicdomain/zero/1.0/) applies to the data made available in this article, unless otherwise stated in a credit line to the data. 
Conclusions: Our review suggested that future single study-based economic evaluations of pharmacogenetic testing should report uncertainty analysis, as this could significantly affect the robustness of economic evaluation results. A specific ranking system for the quality of evidence is needed for the economic evaluation of pharmacogenetic testing of ADRs. Differences in parameters, methods and outcomes across studies, as well as population-level and system-level differences, may lead to the difficulty of comparing cost-effectiveness results across countries.

Keywords: Pharmacogenomics, Adverse drug reactions, Economic-evaluation, Systematic review, Personalized medicine

\section{Background}

In the United States (US), adverse drug reactions (ADRs) are the fourth to the sixth leading cause of death, with approximately more than 100,000 deaths per year [1]. Besides this, there is a similar trend in Europe, with approximately $5 \%$ of all hospitalizations and 197,000 deaths annually reported [2, 3]. The most severe lifethreatening ADRs are the Stevens-Johnson syndrome (SJS) and toxic epidermal necrolysis (TEN). The majority of cases are caused by reactions to certain drugs, e.g., allopurinol, sulfa-drugs or carbamazepine. Moreover, ADRs could result in substantial economic burden. The annual economic impact of severe and fatal ADRs leading to mortality and morbidity was found to be exceptionally high, totaling nearly $\$ 177$ billion in the US and $€ 79$ billion in Europe [2, 4].

Nowadays, there are several methods for investigating people who are at risk of ADRs according to clinical features, such as renal or liver function, age, dosage of administration, as well as identification of any drug interaction. Genetic factors can also be the cause of ADRs, which accounted for approximately $10-20 \%$ [5]. Genetic information obtained from polymorphism-based pharmacogenomics or pharmacogenetics is highly crucial to better identifying responders and non-responders to medications, as well as people who are at risk of ADRs or drug inefficacy prior to prescription [6]. Moreover, there has been an increasing number of genetic associations to develop clinically useful tests through international guidelines, including the Clinical Pharmacogenetics Implementation Consortium (CPIC), the Royal Dutch Association for the Advancement of Pharmacy - Pharmacogenetics Working Group (DPWG) and the Canadian Pharmacogenomics Network for Drug Safety (CPNDS).

The CPIC has developed guidelines on evidence-based pharmacogenetic testing for 54 drugs-gene pairs, which supported and guided the translation of clinically relevant aspect. In addition to this, pharmacogenetics-based therapeutic recommendations for 94 and 8 drugs-gene pairs were published by DPWG and the CPNDS, respectively [7]. It is important to note that the application of pharmacogenetic information before prescribing the corresponding medication is beneficial to avoid serious ADRs or to guide genotype-specific dosing, thereby enhancing the effective use of drug treatment. Therefore, national drug agencies have approved drug labels containing pharmacogenetic information. As of August 1, 2020, 335, 134, 105 and 52 drug labels were approved by the United States Food and Drug Administration (US FDA), the European Medicines Agency (EMA), the US Health Care Service Corporation (HCSC) and the Pharmaceuticals and Medical Devices Agency (PMDA) of Japan, respectively $[6,7]$.

Currently, the most important criteria for its implementation in clinical practice is not only clinical evidence of pharmacogenetic testing, but also its value for money, which can be proved by an economic evaluation being a vital tool used to inform resource allocation in the decision-making process, especially in developed countries $[8,9]$. Therefore, the quality of methodological rigor in cost-effectiveness studies is required to increase the reliability of such studies. Until now, there were two systematic reviews specifically focusing on economic evaluation of pharmacogenetic testing to prevent ADRs $[10,11]$. The first review published in 2008, [10] was aimed at determining the cost of thiopurine methyltransferase (TPMT) genotyping per averted case of neutropenia. It did not, however, evaluate the quality of included studies. Later in 2016, another review, [11] which included all studies up to 2015, assessed the quality of studies in terms of their reporting and evidence of clinical effectiveness of testing but did not include other parameters. Notably, it has been suggested that the sources of evidence for clinical effectiveness, baseline clinical value, resource utilization, cost and utility data, all of which can influence and contribute to biased estimates of economic evaluation results, should be taken into account [12].

Therefore, this review aimed to update a systematic review and critically appraise the quality of existing economic evaluations of pharmacogenetic testing to prevent ADRs, in terms of reporting and sources of evidence used for all significant model inputs, such as clinical effectiveness, baseline clinical data, resource use, cost and utilities. Due to methodological differences across 
studies, as well as population-level and system-level differences, our findings could assist in identifying the potential model parameters that could influence the cost-effectiveness results and their transferability across geographic regions. They could also be valuable in a future and robust cost-effectiveness analysis of pharmacogenetic testing to prevent ADRs, which might help policy-makers make better decisions on allocating resources effectively and implement such testing into clinical practice.

\section{Methods}

A systematic review protocol was initially registered with PROSPERO, an international prospective registry of systematic reviews (identification number CRD42019142060) and available from: http://www. crd.york.ac.uk/PROSPERO/display_record.php?ID= CRD42019142060. The present systematic review was conducted in accordance with the guidelines of the Preferred Reporting Items for Systematic Reviews and Meta-Analysis (PRISMA) [13].

\section{Identification of studies}

We conducted a systematic search in Medline (via PubMed), Scopus and the Centre for Reviews and Dissemination (CRD)'s National Health Service Economic Evaluation Database (NHS EED) to identify relevant studies up to October 2019. The search terms were constructed based on the PICOS domains (patient, intervention, comparison, outcome and study type). The search terms were comprised of the domains on the intervention (pharmacogenetic testing and ADRs) and study type (economic evaluation). There were no restrictions in the domains of patients, comparators and outcomes. The search terms were explicitly used for each search engine and search strategies for each database, as stated in the online appendices (Electronic Supplementary Material) Table A1. The reference lists of the retrieved studies were also explored to identify further studies. The search was updated every six months.

\section{Selection of studies}

Two reviewers (ST and OR) independently selected studies by screening titles and abstracts of all articles based on the eligibility criteria. Full texts of articles identified in the initial screening were retrieved. The studies were included if they met all of the following criteria. First, studies were included if they investigated pharmacogenetic testing of human genetic variations, which guided drug therapies to prevent ADRs. Second, the study type was an economic evaluation, e.g., costeffectiveness analysis (CEA), cost-utility analysis (CUA), cost-benefit analysis (CBA) or cost-minimization analysis (CMA). Studies were excluded if the drug and pharmacogenetic testing were not on the list of the available clinical practice guidelines (e.g., CPIC, DPWG, CPNDS), or if the prescribing information for labelling was not approved by the US FDA as of August 1, 2020. Any disagreements were resolved through discussion.

\section{Data extraction}

Data were extracted independently by two authors (ST and OR) using a data extraction form, which included the study characteristics, author, year of publication, setting, target populations, intervention, comparator, marker frequency, methods, perspective, time horizon, discounting, uncertainty analysis, and outcome measures, in terms of incremental cost, incremental cost per quality adjusted life year (QALY) or life year (LY) gained or cost per adverse reaction/event avoided. We also gathered the parameters, which may affect the costeffectiveness results according to the uncertainty analysis results of individual studies.

\section{Quality assessment of economic evaluation reporting}

The quality of economic evaluation reporting was appraised using the Consolidated Health Economic Evaluation Reporting Standards (CHEERS) checklist with 24 items [14]. Two independent authors (ST and OR) assessed the quality of reporting and any disagreements were resolved through discussion. A percentage of agreement and disagreement by checklist item was calculated. We evaluated the quality of reporting along with the CHEERS checklist by rating scores as follows: the study met all standards (score $=1)$, the study met some standards (score $=0.5$ ), the study did not meet the standards $($ score $=0)$, or the study was not applicable (N/A). For instance, in the checklist item indicating whether the study reported time horizon and described its appropriateness, a score of $1,0.5$ and 0 will be given if the authors met all standards (i.e., they reported both the time horizon and reason why it was appropriate), if they met some standards (i.e., they reported either the time horizon or description of its appropriateness), and if they did not meet the standards (i.e., failed to report both).

\section{Quality assessment of evidence used}

The quality of evidence for input parameters used in economic evaluations, such as clinical effect sizes, baseline clinical data, resource use, costs and utilities (for cost-utility analyses) was assessed using the hierarchy of data sources developed by Cooper et al. [12]. Each item was evaluated and given a rank ranging from 1 to 6 , and 9 was applied to a source which was not clear. For example, for parameters related to clinical effect sizes, rank $1+$ or 1 was given if the data were obtained from a meta-analysis of randomized controlled trials (RCTs) or single RCT with direct comparison measuring final 
outcomes, respectively. Other rates included: rank 2 (a single RCT with direct comparison measuring surrogate outcomes), rank 3 (a single placebo RCTs measuring surrogate outcomes), rank 4 (case control or cohort studies), rank 5 (case report or case series) and rank 6 (expert opinion). Two authors (ST and OR) independently assessed and ranked data sources of the input parameters based on the hierarchy of data sources published by Cooper et al. [12]. Any disagreements were resolved through discussion and a percentage of agreement and disagreement by input parameter was calculated.

Transferability assessment of economic evaluation studies We applied the transferability method developed by Welte et al. [15] to identify potential transferability factors across countries, which can be categorized into three groups: (1) methodology (i.e., perspective, time horizon, cost categories, and discount rate) (2) healthcare system (i.e., practice variation and technology availability), and (3) population characteristics (i.e., disease incidence/prevalence, life expectancy, acceptance and compliance). Based on our review, economic evaluation studies of pharmacogenetic testing conducted across countries were selected as a case-study to assess whether different transferability factors could directly affect the difference in costs and outcomes of the economic evaluation results.

\section{Results}

\section{Search results}

A total of 6718 studies were searched from Medline (1544 studies), Scopus (3010 studies) and Cochrane Database of Systematic Reviews (CDSR) (2164 studies). After excluding 816 duplicates, 5902 studies were screened for titles and abstracts. From these, 5824 studies were excluded for several reasons. The most common reasons were "non-genetic interventions" and "nondrug-related ADRs", as described in Fig. 1. A total of 64 studies met the inclusion criteria. Nevertheless, five studies were excluded since they were not included in the list of available clinical practice guidelines. Finally, 59 studies were eligible for data extraction.

\section{Characteristics of the included studies}

The general characteristics of all included studies are presented in online appendices (Electronic Supplementary Material) Table A2. All studies were published between 2002 and 2018. CUA was the most frequent type of economic evaluation (41 studies) (70\%), followed by CEA (10 studies) (17\%), CBA (5 studies) (8\%), and then CMA (3 studies) (5\%). Table 1 demonstrates the number of studies categorized by therapeutic area-gene and ADRs, as well as by region. Majority of the studies were conducted in European and American countries (43 studies) (73\%), while studies related to $H L A-B * 58: 01$ allopurinol and $H L A-B * 15: 02$-carbamazepine were mostly found in Asian countries. Most studies investigated the therapeutic area of cardiovascular diseases (24 studies) [16-39], followed by gout (8 studies) [40-47], human immunodeficiency virus (HIV) infection (8 studies) [48-55], autoimmune diseases (8 studies) [56-63], and epilepsy/neuropathic pain (6 studies) [64-69], cancer (3 studies) [70-72], major depressive disorder [73], and hormone replacement therapy [74].

The majority of pharmacogenetic testing to prevent ADRs were CYP2C9 and VKORC1 testing before prescription of warfarin (14 studies) [16-29], CYP2C19 genotype screening for selection of antiplatelet therapy (i.e., clopidogrel) after percutaneous coronary intervention (PCI) for acute coronary syndrome (ACS) patients (9 studies) [30-38], and $H L A-B * 58: 01$ screening before prescribing allopurinol in patients with gout (8 studies) [40-47]. Moreover, the severity of ADRs related to gene-drug pairs was grouped into two major types: severe ADRs (life-threatening or fatal ADRs) and common ADRs. Pharmacogenetic testing and drugs associated with severe ADRs were $H L A-B * 58: 01$-allopurinol induced SJS/TEN/drug reaction with eosinophilia and systemic symptoms (DRESS), HLA-B*57:01-abacavir induced hypersensitivity reaction, $H L A-B * 15: 02$ and $H L A-A * 31: 01$ - carbamazepine induced SJS/TEN/hypersensitivity, TPMT-azathioprine induced severe bone marrow toxicity, UGT1A1- irinotecan induced severe neutropenia and DPYD- fluoropyrimidines induced severe hematologic/GI toxicity. Meanwhile, the others were pharmacogenetic testing and drug-associated common ADRs. Most of the genetic information regarding gene-drug pairs was published by the CPIC guideline [75] and drug labels were approved by the US FDA, except for the study determining Factor V Leiden screening before receiving estrogen combined with oral contraceptives [76].

\section{Quality assessment of economic evaluation reporting using the CHEERS checklist}

The quality of economic evaluation reporting using the CHEERS checklist [14] is summarized in online appendices (Electronic Supplementary Material) Table A3. All included studies clearly described the study population, measurement of effectiveness based on single study or synthesis estimated, as well as approaches for estimating resource use and costs with the percentage of agreement between two independent authors ranging from 81 to $100 \%$, indicating that the score rating for each item was reliable. In contrast, only $22 \%$ of the single (trial) study-based performed uncertainty analysis of the parameters and evaluated their effects, as detailed in 


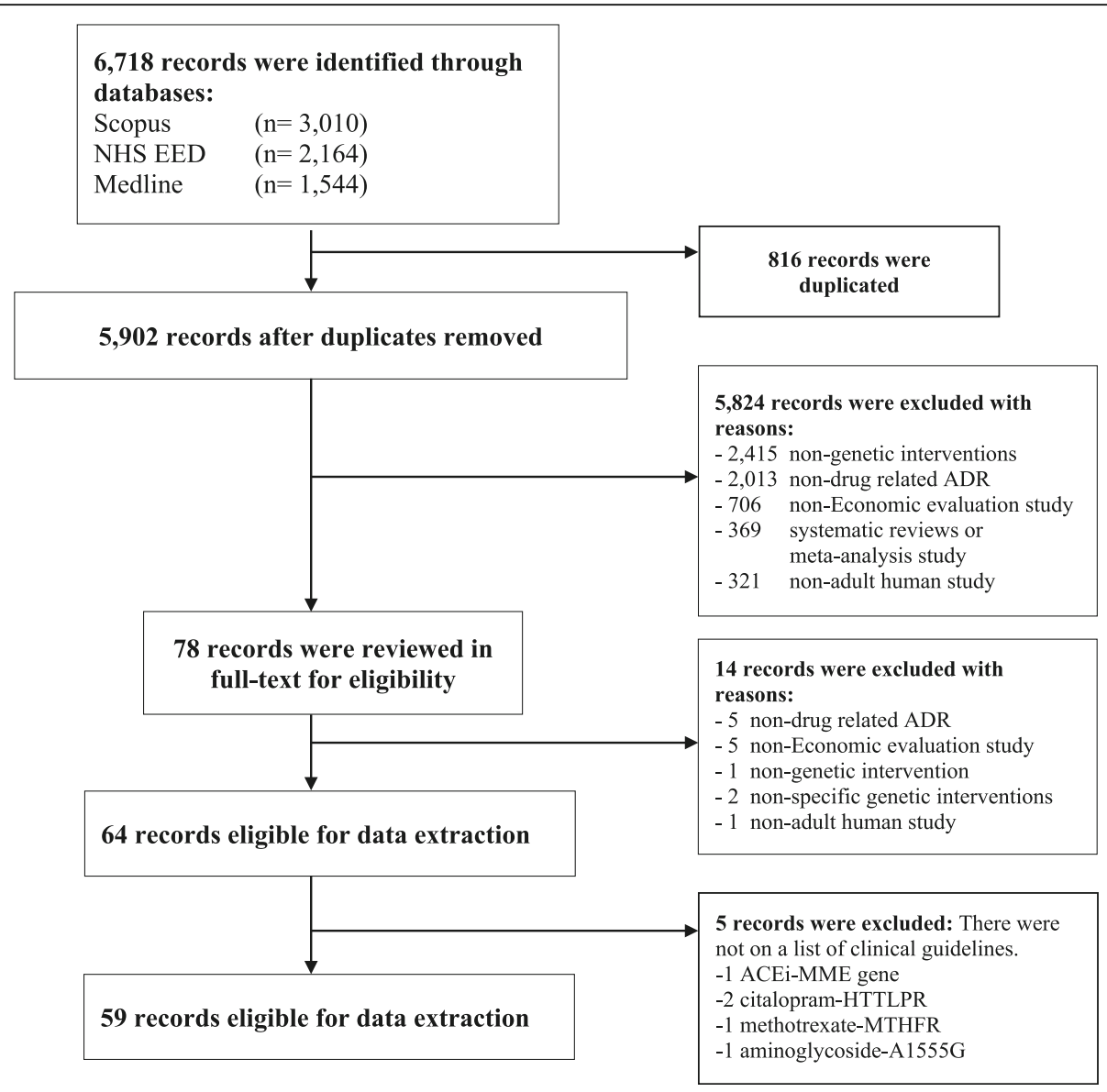

Fig. 1 PRISMA flow of study selection process

Table 2. Notably, 18 studies (31\%) adopted a health service or healthcare payer's viewpoint in the analysis, while 10 studies (17\%) and 9 studies (15\%) presented societal and healthcare system's perspectives, respectively. There were nine studies (15\%) that did not mention the study's perspective $[27,28,32,41,49$, $57,59,66,74]$. The time horizon used for cost and consequence evaluation ranging from six weeks to a lifetime was also reported in the above studies, while five studies (8\%) did not state the time horizon [32, 48, 61, 66, 70]. Among the studies that specified a time horizon exceeding one year, there were seven studies $(18 \%)$ that did not report the discount rate for costs and outcomes [32, 39, 48, 49, 61, 66, 70].

For clinical effectiveness data, 50 studies (85\%) used a single, study-based estimate and nine studies (15\%) used synthesis-based estimates. All studies clearly described the source of evidence. Most studies (50 studies; 85\%) were conducted based on the decision-analytic model, while nine studies (15\%) used a single study-based economic evaluation. Seven studies (78\%) including retrospective, observational, or RCT-based economic evaluations studies did not mention uncertainty analysis $[27,32,41,48,59,61,66]$ as they did not indicate confidence intervals denoting uncertainty measures. However, all model-based economic evaluations performed uncertainty analysis. More than half (26 of 50 model-based studies; $52 \%$ ), performed both one-way sensitivity analysis and probabilistic sensitivity analysis (PSA), while some conducted the one-way sensitivity analysis only (6 studies; $12 \%$ ) and PSA only (6 studies; $12 \%$ ). There were 12 studies $(20 \%)$ and 13 studies $(22 \%)$ that did not describe any source of funding $[21,28,40,41,48,51,54$, $57,59-62]$ and the potential for conflict of interest of study contributors $[29,37,43,45,52,54,57,59-61,63$, $71,74]$, respectively.

\section{Quality assessment of evidence used}

Only 16 studies (27\%) obtained clinical effectiveness data of testing from high-quality evidence (a single RCT with direct comparison, rank 1), while about half (49\%) retrieved evidence from case-control or cohort studies (rank 4). Nevertheless, 8 studies (14\%) used clinical effectiveness data of testing from the meta-analysis of 
Table 1 Number of studies classified by therapeutic area-gene and ADRs and by region

\begin{tabular}{|c|c|c|c|c|c|c|c|c|}
\hline \multicolumn{4}{|c|}{ Therapeutic area-gene and ADRs } & \multirow[t]{2}{*}{$\begin{array}{l}\text { Clinical } \\
\text { guideline }\end{array}$} & \multirow{2}{*}{$\begin{array}{l}\text { FDA } \\
\text { approved } \\
\text { labelling }\end{array}$} & \multicolumn{3}{|c|}{$\begin{array}{l}\text { Number of studies by } \\
\text { region }\end{array}$} \\
\hline Drug & Gene & ADRs & $\begin{array}{l}\text { Severity of } \\
\text { ADRs }\end{array}$ & & & Asians & $\begin{array}{l}\text { Europeans/ } \\
\text { USA }\end{array}$ & Total \\
\hline \multicolumn{9}{|l|}{ Cardiovascular disease (24) } \\
\hline Warfarin & $\begin{array}{l}\text { CYP2C9 and } \\
\text { VKORC1 }\end{array}$ & bleeding & NS & $\begin{array}{l}\text { CPIC, DPWG, } \\
\text { CPNDS }\end{array}$ & $\sqrt{ }$ & 2 & 12 & 14 \\
\hline Clopidogrel & CYP2C19 & $\begin{array}{l}\text { major cardiac/adverse } \\
\text { CV events }\end{array}$ & NS & CPIC,DPWG & $\sqrt{ }$ & 1 & 8 & 9 \\
\hline Statins & $\begin{array}{l}\text { Pharmacogenetics } \\
\text { test }\end{array}$ & myopathy & NS & CPIC, DPWG & $\sqrt{ }$ & - & 1 & 1 \\
\hline \multicolumn{9}{|l|}{ Gout (8) } \\
\hline Allopurinol & $H L A-B * 58: 01$ & SJS/TEN, DRESS & S & CPIC & $\sqrt{ }$ & 6 & 2 & 8 \\
\hline \multicolumn{9}{|l|}{ HIV infection (8) } \\
\hline Abacavir & $H L A-B^{*} 57: 01$ & Hypersensitivity & S & CPIC, DPWG & $\sqrt{ }$ & 1 & 6 & 7 \\
\hline Efavirenz & CYP2B6 & CNS toxicity & NS & CPIC,DPWG & $\sqrt{ }$ & - & 1 & 1 \\
\hline \multicolumn{9}{|l|}{ Autoimmune disease (8) } \\
\hline Azathioprine & TPMT & $\begin{array}{l}\text { severe bone marrow } \\
\text { toxicity }\end{array}$ & S & CPIC, DPWG & $\sqrt{ }$ & 1 & 7 & 8 \\
\hline \multicolumn{9}{|l|}{ Epilepsy/neuropathic pain (6) } \\
\hline Carbamazepine & $H L A-B^{*} 15: 02$ & SJS/TEN & S & CPIC, CPNDS & $\sqrt{ }$ & 5 & - & 5 \\
\hline Carbamazepine & $H L A-A^{*} 31: 01$ & $\begin{array}{l}\text { SJS/TEN, } \\
\text { Hypersensitivity }\end{array}$ & S & CPIC, CPNDS & $\sqrt{ }$ & - & 1 & 1 \\
\hline \multicolumn{9}{|l|}{ Cancer (3) } \\
\hline Irinotecan & UGT1A1 & severe neutropenia & S & DPWG & $\sqrt{ }$ & - & 2 & 2 \\
\hline Fluoropyrimidines & DPYD & $\begin{array}{l}\text { severe hematologic, } \\
\text { Gl toxicity }\end{array}$ & S & CPIC, DPWG & $\sqrt{ }$ & - & 1 & 1 \\
\hline \multicolumn{9}{|l|}{ Major depressive disorder (1) } \\
\hline Nortriptyline & CYP2D6 & $\begin{array}{l}\text { anticholinergic } \\
\text { symptoms }\end{array}$ & NS & CPIC, DPWG & $\sqrt{ }$ & - & 1 & 1 \\
\hline \multicolumn{9}{|c|}{ Hormone replacement therapy (1) } \\
\hline $\begin{array}{l}\text { Estrogen combined in oral } \\
\text { contraceptives }\end{array}$ & Factor V Leiden & $\begin{array}{l}\text { venous } \\
\text { thromboembolic } \\
\text { disease }\end{array}$ & NS & DPWG & - & - & 1 & 1 \\
\hline
\end{tabular}

CBA: cost-benefit analysis, CEA: cost-effectiveness, CMA: cost-minimization analysis, CUA: cost-utility analysis, CPIC: the Clinical Pharmacogenetics Implementation Consortium, CPNDS: the Canadian Pharmacogenomics Network for Drug Safety, DPWG: the Royal Dutch Association for the Advancement of Pharmacy - Pharmacogenetics Working Group, DRESS: drug reaction with eosinophilia and symptomatic symptoms, FDA: the United States Food and Drug Administration, NS: Non-Severe ADRs, S: Severe ADRs, SJS: Stevens-Johnson syndrome, TEN: toxic epidermal necrolysis

case-control study with direct comparison, which was not listed in the hierarchy of data sources by Cooper et al. [12]. Moreover, only four (7\%) and one studies (2\%) applied the baseline clinical data from a highquality evidence [case series specifically performed for the study (rank 1) and the analysis of administrative databases including only patients in interested settings (rank 2)]; whereas most studies (85\%) obtained clinical effectiveness data of testing from old case series, analysis of reliable administrative databases, or estimated from RCTs (rank 4). On the other hand, most studies sourced resource use and cost information from a high-quality evidence [prospective data analysis conducted for specific study (rank 1) or recently published cost estimation based on reliable databases (rank 2)], except for one study on $H L A-B * 57: 01$-abacavir, which used data from expert opinions (rank 6) [52]. For CUA studies, the utility data were mostly (93\%) estimated from a direct utility from a previous study in patients with the disease of interest (rank 3). Only one study for CYP2B6-efavirenz did not define the data source (rank 4) [53]. However, there was no evidence obtained from indirect utility or expert opinion. Overall, the percentage of agreement between two independent authors ranging from 81 to $97 \%$, suggesting that the ranking of data sources was reliable, see Table 3. 
Table 2 Quality assessment results of economic evaluation reporting using the CHEERS checklist

\begin{tabular}{|c|c|c|c|}
\hline Item (Item No) & $\begin{array}{l}\text { Number of studies met the } \\
\text { recommendations }\end{array}$ & $\begin{array}{l}\text { Percentage } \\
(\%)\end{array}$ & $\%$ Agreement $†$ \\
\hline Target population and subgroups (4) & $59 / 59$ & 100 & 98 \\
\hline Study perspective (6) & $50 / 59$ & 85 & 81 \\
\hline Comparators (7) & $58 / 59$ & 98 & 98 \\
\hline Time horizon (8) & $54 / 59$ & 92 & 98 \\
\hline Discount rate for costs and outcomes $(9)^{\dagger+}$ & $32 / 39$ & 82 & 86 \\
\hline $\begin{array}{l}\text { Measurement of effectiveness (Single study-based estimates) } \\
(11 \mathrm{a})^{\#}\end{array}$ & $50 / 50$ & 100 & 95 \\
\hline 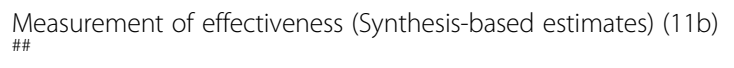 & $9 / 9$ & 100 & 95 \\
\hline Estimating resources and costs (Single study-based EE) $(13 a)^{*}$ & 9/9 & 100 & 95 \\
\hline Estimating resources and costs (Model-based EE) $(13 b)^{* *}$ & $50 / 50$ & 100 & 95 \\
\hline Study parameters (18) & $50 / 59$ & 85 & 84 \\
\hline Incremental costs and outcomes (19) & $57 / 59$ & 97 & 95 \\
\hline Characterizing uncertainty (Single study-based EE) $(20 a)^{*}$ & $2 / 9$ & 22 & 98 \\
\hline Characterizing uncertainty (Model-based EE) (20b)** & $50 / 50$ & 100 & 100 \\
\hline Source of funding (23) & $47 / 59$ & 80 & 95 \\
\hline Conflicts of interest (24) & $46 / 59$ & 78 & 97 \\
\hline
\end{tabular}

EE: Economic evaluation

† Percent agreement between two independent raters

t† The denominator for the calculations is the studies that reported the discount rate for costs and/or outcomes were from a study period longer than one year, including 39 studies

\# The denominator for the calculations is a single study-based estimate of clinical effectiveness data, including 50 studies

\#\# The denominator for the calculations is synthesis-based estimates of clinical effectiveness data, including 9 studies

* The denominator for the calculations is a single study-based economic evaluation, including 9 studies

** The denominator for the calculations is a single model-based economic evaluation, including 50 studies

\section{Cost-effectiveness results}

In terms of pharmacogenetic testing and drugs associated with ADRs in particular disease areas, such as cardiovascular diseases, gout, HIV infection, autoimmune diseases, epilepsy/neuropathic pain, cancer, major depressive disorder, and hormone replacement therapy, the results of economic evaluation studies are summarized and presented in Table 4.

\section{Cardiovascular diseases}

CYP2C9 and VKORC1 and warfarin-induced risk of bleeding There were 14 economic evaluation studies of CYP2C9 and VKORC1 testing before prescription of warfarin to prevent risks of bleeding. Over the prior from 2004 to 2017, these studies were conducted in Korea (1 study) [16], UK and Sweden (1 study) [17], Croatia (1 study) [18], Thailand (1 study) [21], Sweden (1 study) [20], Netherlands [28] and US (8 studies) [19, 22-27, 29]. Ten studies were CUA with model-based economic evaluation [16-19, 21-26] and another study was CUA with a trial-based [20] economic evaluation to estimate resource use associated with the interventions. Two studies were CEA with model-based $[28,29]$ and one study was CEA based on a retrospective study [27]. Seven studies were explicitly conducted in patients with atrial fibrillation and one study investigated mechanical heart valve replacement (MHVR). The rest of the studies were used in newly initiated warfarin therapy. Nine studies showed that CYP2C9 and VKORC1 testing to prevent the risk of bleeding would be a cost-effective intervention [16-20, 22, 27-29] (i.e., less costly and more effective than treatment without genotyping). One study suggested that testing would be cost-effective if it increased the time spent in the target international normalized ratio (INR) range during the first three months of treatment by 5 to $9 \%$ [25]. Nevertheless, four studies from Thailand [21] and US [23, 24, 26] suggested that those testings would not be cost-effective due to the effectiveness of testing in reducing out-of-range INR values.

CYP2C19 and clopidogrel-induced major adverse cardiovascular events (MACE) There were nine studies which conducted an economic evaluation of CYP2C19 testing before prescription of clopidogrel to avoid cardiovascular events in patients with ACS undergoing PCI. They were performed in Hong Kong (1 study) [30], 
Table 3 Quality assessment results of evidence used based on the hierarchy of data sources by Cooper et al. [12]

\begin{tabular}{|c|c|c|c|c|c|c|c|c|c|}
\hline \multirow[t]{2}{*}{ Input parameters } & \multicolumn{8}{|c|}{ Hierarchy of data sources } & \multirow{2}{*}{$\begin{array}{l}\% \\
\text { Agreement }\end{array}$} \\
\hline & 1 & 2 & 3 & 4 & 5 & 6 & 9 & $\#$ & \\
\hline Clinical effect sizes & $16(27 \%)$ & 0 & 0 & $29(49 \%)$ & 0 & $2(3 \%)$ & $4(7 \%)$ & $8(14 \%)$ & 85 \\
\hline Baseline clinical data & $4(7 \%)$ & $1(2 \%)$ & 0 & $51(85 \%)$ & $1(2 \%)$ & $1(2 \%)$ & $1(2 \%)$ & & 81 \\
\hline Resource use & $7(12 \%)$ & $51(86 \%)$ & 0 & 0 & 0 & $1(2 \%)$ & 0 & & 92 \\
\hline Costs & $6(10 \%)$ & $53(90 \%)$ & 0 & 0 & 0 & 0 & 0 & & 85 \\
\hline Utility & $2(5 \%)$ & 0 & $38(93 \%)$ & $1(2 \%)$ & 0 & 0 & 0 & & 97 \\
\hline
\end{tabular}

\# Meta-analysis of case-control with direct comparison between comparator therapies and measuring final outcomes

*Percentage of agreement between two independent raters

Netherlands (1 study) [32], Australia (1 study) [35], New Zealand (1 study) [38] and US (5 study) [31, 33, 34, 36, 37]. They were conducted from 2012 to 2018. Seven studies were CUA with model-based approach [30, 31, 33-36, 38] and one study was CUA with trial-based [32] Others were CEA with model-based [37] economic evaluation. All studies showed that CYP2C19 testing would be a potentially cost-effective treatment strategy for avoiding MACE. Nevertheless, some studies considered ticagrelor and/or prasugrel as alternative drugs with a higher cost than clopidogrel for those who tested positive.

Pharmacogenetic testing and statin-induced myopathy One study developed testing that identified statin-induced myopathy in cardiovascular patients by using CUA with model-based economic evaluation in 2017. The results demonstrated that genotyping would be a cost-effective intervention, with a testing cost of CAN\$ 906 that was less than the cost of no testing in Canada [39].

\section{Gout}

$H L A-B * 58: 01$ and allopurinol-induced SJS/TEN and DRESS There were eight economic evaluation studies of $H L A-B * 58: 01$ screening before prescribing allopurinol in gout patients to prevent SIS/TEN and DRESS) that were performed in China (1 study) [41], Malaysia (1 study) [40], US (1 study) [44], Taiwan (1 study) [43], UK (1 study) [42], Singapore (1 study) [46], Korea (1 study) [45], and Thailand (1 study) [47] from 2014 to 2018. Six studies used CUA with model-based economic evaluation [40, 42-44, 46, 47]), one performed CMA with trial-based [41] and one conducted CBA with modelbased approach [45]. All studies were conducted in patients with gout, and two studies were explicitly conducted in gout patients with Chronic Kidney Disease (CKD) $[43,45]$. Most of the studies considered allopurinol-induced SJS/TEN, but only one study considered both SJS/TEN and DRESS. Five studies applied febuxostat as an alternative drug in the model [41-45] to patients who tested positive with $H L A-B * 58: 01$. However, probenecid has been used in Malaysia, Thailand and Singapore $[40,46,47]$ as febuxostat is not regularly used in the usual clinical practice.

Three studies showed that $H L A-B * 58: 01$ genotyping would be a cost-effective in China, Taiwan and Thailand [41, 43, 47] and cost-saving intervention in the Korean study [45]. Nevertheless, three studies from Malaysia [40], UK [42] and Singapore [46] suggested that HLA$B * 58: 01$ genotyping would not be cost-effective as the cost of the pharmacogenomics testing and alternative drugs, such as febuxostat were too high, and the efficacy of alternative drugs was less than that of allopurinol (e.g., probenecid). Moreover, the study in US [44] showed that genotyping would be cost-effective for Asians and African Americans. However, it would not be cost-effective for Caucasians or Hispanics. Therefore, the incremental cost-effectiveness ratios (ICERs) might vary substantially across racial or ethnic groups, following by their $H L A-B * 5801$ frequency.

\section{HIV infection}

$H L A-B * 57: 01$ and abacavir-induced hypersensitivity reaction There were seven economic evaluation studies of $H L A-B * 57: 01$ screening before prescribing abacavir for HIV positive patients to prevent hypersensitivity reactions (HSR) that were conducted in Russia (1 study) [48], Singapore (1 study) [49], Spain (1 study) [51], Germany (1 study) [50], UK (1 study) [54], and US (2 studies) [52, 53] from 2004 until 2018. Most studies were conducted in Europe and US [48, 50-54], and only one study investigated the Asian population [49]. Results showed that allele frequencies in Europe and US were much higher (ranged from 3.7-7.3\%) than those of the Asian population (1.1\% in Han Chinese, $1.8 \%$ in Malays), except for Indians (3.6\%). Three studies were CUA with model-based [49, 52, 53], two studies were CEA with model-based [51, 54] and one was CBA with modelbased economic evaluation [50]. The rest was CMA based on a retrospective study [48]. Four studies in Russia, Germany, UK and US demonstrated that HLA- 
Table 4 Cost-effectiveness results of included studies

\begin{tabular}{|c|c|c|c|c|c|c|}
\hline No & $\begin{array}{l}\text { Author, Year of } \\
\text { published }\end{array}$ & $\begin{array}{l}\text { study } \\
\text { setting }\end{array}$ & $\begin{array}{l}\text { Target } \\
\text { populations }\end{array}$ & Intervention vs comparator & $\begin{array}{l}\text { Cost } \\
\text { effectiveness } \\
\text { threshold }\end{array}$ & $\begin{array}{l}\text { Cost-effectiveness results } \\
\text { (ICER/ICUR) }\end{array}$ \\
\hline \multicolumn{7}{|c|}{$\begin{array}{l}\text { Drug: warfarin, Biomarker: CYP2C9 and VKORC1 } \\
\text { ADRs: bleeding events }\end{array}$} \\
\hline 1 & $\begin{array}{l}\text { Kim,DJ et al., } \\
2017 \text { [16] }\end{array}$ & Korea & $\begin{array}{l}\text { mechanical } \\
\text { heart valve } \\
\text { replacement } \\
\text { (MHVR) }\end{array}$ & $\begin{array}{l}\text { (a) warfarin } \\
\text { (b) CYP2C19 and VKORC1 } \\
\text { genotyping-guided dosing of } \\
\text { warfarin }\end{array}$ & $\begin{array}{l}\$ 50,000 \text { per } \\
\text { QALY gained }\end{array}$ & $\begin{array}{l}\text { - ICER (b) vs (a): } \$ 13,562 \text { per QALY } \\
\text { gained }\end{array}$ \\
\hline 2 & $\begin{array}{l}\text { Verhoef et al., } \\
2016 \text { [17] }\end{array}$ & $\begin{array}{l}\text { UK and } \\
\text { Sweden }\end{array}$ & $\begin{array}{l}\text { Atrial } \\
\text { Fibrillation (AF) }\end{array}$ & $\begin{array}{l}\text { (a) warfarin } \\
\text { (b) CYP2C19 and VKORC1 } \\
\text { genotyping-guided dosing of } \\
\text { warfarin }\end{array}$ & $\begin{array}{l}\text { UK } £ 20,000 \text { per } \\
\text { QALY gained, } \\
\text { Sweden } 500,000 \\
\text { SEK }\end{array}$ & $\begin{array}{l}\text { - In UK: ICER (b) vs (a): £6702 per QALY } \\
\text { gained } \\
\text { - In Sweden: ICER (b) vs (a): 253,848 SEK } \\
\text { per QALY gained }\end{array}$ \\
\hline 3 & $\begin{array}{l}\text { Mitropoulou } \\
\text { et al., } 2015[18]\end{array}$ & Croatia & $\begin{array}{l}\text { ischemic } \\
\text { stroke patients } \\
\text { with AF }\end{array}$ & $\begin{array}{l}\text { (a) warfarin } \\
\text { (b) CYP2C19 and VKORC1 } \\
\text { genotyping-guided dosing of } \\
\text { warfarin }\end{array}$ & $\begin{array}{l}€ 40,000 \text { to } \\
€ 50,000 \text { per } \\
\text { QALY gained }\end{array}$ & $\begin{array}{l}\text { - ICER (b) vs (a): €31,225 per QALY } \\
\text { gained }\end{array}$ \\
\hline 4 & $\begin{array}{l}\text { Chong, HYet al, } \\
2014[21]\end{array}$ & Thailand & $\begin{array}{l}\text { newly initiated } \\
\text { warfarin } \\
\text { therapy }\end{array}$ & $\begin{array}{l}\text { (a) warfarin } \\
\text { (b) CYP2C19 and VKORC1 } \\
\text { genotyping-guided dosing of } \\
\text { warfarin }\end{array}$ & $\begin{array}{l}160,000 \text { THB or } \\
\$ 5333 \text { per QALY } \\
\text { gained }\end{array}$ & $\begin{array}{l}\text { - Healthcare system perspective: } \\
\text { ICER (b) vs (a): } 1,477,042 \text { THB }(\$ 49,234) \\
\text { per QALY gained } \\
\text { - Societal perspective } \\
\text { ICER (b) vs (a): } 1,473,852 \text { THB }(\$ 49,128) \\
\text { per QALY gained }\end{array}$ \\
\hline 5 & $\begin{array}{l}\text { You, et al., } 2014 \\
\text { [19] }\end{array}$ & USA & AF & $\begin{array}{l}\text { (a) warfarin } \\
\text { (b) CYP2C19 and VKORC1 } \\
\text { genotyping-guided treatment }\end{array}$ & $\begin{array}{l}\$ 50,000 \text { per } \\
\text { QALY gained }\end{array}$ & - ICER (b) vs (a): \$ 2843 per QALY gained \\
\hline 6 & $\begin{array}{l}\text { Pink et al., } 2014 \\
\text { [20] }\end{array}$ & Sweden & $\begin{array}{l}\text { Non-valvular } \\
\text { AF }\end{array}$ & $\begin{array}{l}\text { (a) warfarin } \\
\text { (b) CYP2C19 and VKORC1 } \\
\text { genotyping-guided treatment } \\
\text { (c) dabigatran } \\
\text { (d) rivaroxaban } \\
\text { (e) apixaban }\end{array}$ & $\begin{array}{l}£ 20,000-30,000 \\
\text { per QALY } \\
\text { gained }\end{array}$ & $\begin{array}{l}\text { - ICER (b) vs (a): } £ 13,226 \text { per QALY } \\
\text { gained } \\
\text { - ICER (e) vs (b): } £ 20,671 \text { per QALY } \\
\text { gained } \\
\text { - (d) is dominated by (c) and (e), high } \\
\text { cost and lower QALYs than (c) and (e) } \\
\text { - (c) is dominated by (e), high cost and } \\
\text { lower QALYs than (e) }\end{array}$ \\
\hline 7 & $\begin{array}{l}\text { You et al., } 2012 \\
\text { [22] }\end{array}$ & USA & $\begin{array}{l}\text { newly } \\
\text { diagnosed AF }\end{array}$ & $\begin{array}{l}\text { (a) warfarin } \\
\text { (b) CYP2C19and } \\
\text { VKORC1genotyping-guided } \\
\text { treatment } \\
\text { (c) dabigatran } 110 \mathrm{mg} \text { twice daily } \\
\text { (d) dabigatran } 150 \mathrm{mg} \text { twice daily }\end{array}$ & $\begin{array}{l}\$ 50,000 \text { per } \\
\text { QALY gained }\end{array}$ & $\begin{array}{l}\text { - (a) is dominated by (b), high cost and } \\
\text { lower QALYs than (b) } \\
\text { - ICER (d) Vs (b): } \$ 13,810 \text { per QALY } \\
\text { gained } \\
\text { - (c) is dominated by (d), (d) lower cost } \\
\text { and more effective than (c) }\end{array}$ \\
\hline 8 & $\begin{array}{l}\text { Meckley et al., } \\
2010 \text { [23] }\end{array}$ & USA & $\begin{array}{l}\text { newly initiated } \\
\text { warfarin } \\
\text { therapy }\end{array}$ & $\begin{array}{l}\text { (a) warfarin } \\
\text { (b) CYP2C19 and VKORC1 } \\
\text { genotyping-guided dosing of } \\
\text { warfarin }\end{array}$ & $\begin{array}{l}\$ 50,000 \text { per } \\
\text { QALY gained }\end{array}$ & $\begin{array}{l}\text { - ICER (b) vs (a): \$ 60,725 per QALY } \\
\text { gained }\end{array}$ \\
\hline 9 & $\begin{array}{l}\text { Eckman et al., } \\
2009[26]\end{array}$ & USA & $\begin{array}{l}\text { Non-valvular } \\
\text { AF }\end{array}$ & $\begin{array}{l}\text { (a) warfarin } \\
\text { (b) CYP2C19 and VKORC1 } \\
\text { genotyping-guided dosing of } \\
\text { warfarin }\end{array}$ & $\begin{array}{l}\$ 50,000 \text { per } \\
\text { QALY gained }\end{array}$ & $\begin{array}{l}\text { • ICER (b) vs (a): \$171,750 per QALY } \\
\text { gained }\end{array}$ \\
\hline 10 & $\begin{array}{l}\text { Patrick et al., } \\
2009 \text { [25] }\end{array}$ & USA & $\begin{array}{l}\text { newly } \\
\text { diagnosed AF }\end{array}$ & $\begin{array}{l}\text { (a) warfarin } \\
\text { (b) CYP2C19 and VKORC1 } \\
\text { genotyping-guided dosing of } \\
\text { warfarin }\end{array}$ & $\begin{array}{l}\$ 50,000 \text { per } \\
\text { QALY gained }\end{array}$ & $\begin{array}{l}\text { - ICER (b) vs (a): ICER< } 50,000 \text { per QALY } \\
\text { gained if it increased the time spent in } \\
\text { the target INR range during the first } 3 \\
\text { months of treatment by } 5 \text { to } 9 \\
\text { percentage points }\end{array}$ \\
\hline 11 & $\begin{array}{l}\text { You et al., } 2009 \\
\text { [24] }\end{array}$ & USA & $\begin{array}{l}\text { newly initiated } \\
\text { warfarin } \\
\text { therapy }\end{array}$ & $\begin{array}{l}\text { (a) warfarin } \\
\text { (b) CYP2C19 and VKORC1 } \\
\text { genotyping-guided dosing of } \\
\text { warfarin }\end{array}$ & $\begin{array}{l}\$ 50,000 \text { per } \\
\text { QALY gained }\end{array}$ & $\begin{array}{l}\text { - ICER (b) vs (a): \$ 347,059 per QALY } \\
\text { gained }\end{array}$ \\
\hline 12 & $\begin{array}{l}\text { McWilliam et al., } \\
2008 \text { [27] }\end{array}$ & USA & $\begin{array}{l}\text { newly initiated } \\
\text { warfarin } \\
\text { therapy }\end{array}$ & $\begin{array}{l}\text { (a) warfarin } \\
\text { (b) CYP2C19 and VKORC1 } \\
\text { genotyping-guided dosing of } \\
\text { warfarin }\end{array}$ & N/A & $\begin{array}{l}\text { - Low baseline bleeding: ICER (b) vs (a): } \\
\$ 82,890 \text { per bleeding averted } \\
\text { - Medium baseline bleeding: ICER (b) vs } \\
\text { (a): } \$ 13,589 \text { per bleeding averted } \\
\text { - High baseline bleeding: (b) is } \\
\text { dominated by (a) lower cost than (a) }\end{array}$ \\
\hline
\end{tabular}


Table 4 Cost-effectiveness results of included studies (Continued)

\begin{tabular}{|c|c|c|c|c|c|c|}
\hline No & $\begin{array}{l}\text { Author, Year of } \\
\text { published }\end{array}$ & $\begin{array}{l}\text { study } \\
\text { setting }\end{array}$ & $\begin{array}{l}\text { Target } \\
\text { populations }\end{array}$ & Intervention vs comparator & $\begin{array}{l}\text { Cost } \\
\text { effectiveness } \\
\text { threshold }\end{array}$ & $\begin{array}{l}\text { Cost-effectiveness results } \\
\text { (ICER/ICUR) }\end{array}$ \\
\hline 13 & $\begin{array}{l}\text { Schalekamp } \\
\text { et al., } 2006 \text { [28] }\end{array}$ & $\begin{array}{l}\text { The } \\
\text { Netherlands }\end{array}$ & $\begin{array}{l}\text { newly initiated } \\
\text { warfarin } \\
\text { therapy }\end{array}$ & $\begin{array}{l}\text { (a) warfarin } \\
\text { (b) CYP2C19 and VKORC1 } \\
\text { genotyping-guided dosing of } \\
\text { warfarin }\end{array}$ & $€ 20,000$ & $\begin{array}{l}\text { - ICER (b) vs (a): € } 4233 \text { per bleeding } \\
\text { averted }\end{array}$ \\
\hline 14 & $\begin{array}{l}\text { You et al., } 2004 \\
\text { [29] }\end{array}$ & USA & $\begin{array}{l}\text { newly initiated } \\
\text { warfarin } \\
\text { therapy }\end{array}$ & $\begin{array}{l}\text { (a) warfarin } \\
\text { (b) CYP2C19 and VKORC1 } \\
\text { genotyping-guided dosing of } \\
\text { warfarin }\end{array}$ & N/A & $\begin{array}{l}\text { - ICER (b) vs (a): \$5778 per bleeding } \\
\text { averted }\end{array}$ \\
\hline
\end{tabular}

Drug: clopidogrel, Biomarker: CYP2C19 ADRs: major cardiac/adverse cardiovascular events

1 Wang $Y$ et al. Hong Kong ACS 2018 [30]

undergoing

$\mathrm{PCl}$

(a) clopidogrel

(b) ticagrelor

(c) CYP2C19 testing guided therapy

Test: positive: ticagrelor; Test negative: clopidogrel

2 Jiang, $M$ et al., USA ACS 2017 [31]

undergoing $\mathrm{PCl}$

(a) clopidogrel

(b) prasugrel or ticagrelor

(c) CYP2C19 testing guided therapy

Test: positive: prasugrel or ticagrelor;

Test negative: clopidogrel

3 Deiman BA et al., the ACS 2016 [32] Netherlands undergoing $\mathrm{PCl}$
(a) clopidogrel
(b) prasugrel
(c) ticagrelor
(d) CYP2C19 testing guided therapy
Test: positive: prasugrel or ticagrelor:
Test negative: clopidogrel

4 Kazi DS et al., USA ACS 2014 [34]

undergoing

$\mathrm{PCl}$

(a) clopidogrel

(b) prasugrel

(c) ticagrelor

(d) CYP2C19 testing guided therapy

Test: positive: prasugrel or ticagrelor;

Test negative: clopidogrel

5 Patel et al., 2014 USA [33]

ACS

undergoing $\mathrm{PCl}$

(a) clopidogrel+asprin

(b) prasugrel+aspirin

(c) CYP2C19 testing guided therapy

Test: positive: prasugrel+asprin;

Test negative: clopidogrel+asprin

6 LALA A et al., USA ACS 2013 [36]

undergoing

$\mathrm{PCl}$

(a) clopidogrel

(b) prasugrel

(c) CYP2C19 testing guided therapy

Test: positive: prasugrel;

Test negative: clopidogrel

7 Sorich et al., 2013 Australia [35]

ACS
undergoing
$\mathrm{PCl}$

(a) clopidogrel

(b) ticagrelor

(c) CYP2C19 testing guided therapy

Test: positive: ticagrelor;

Test negative: clopidogrel

8 Panattoni $L$ et al., New 2012 [38] Zealand
ACS (included the four largest ethnic groups)
$\$ 42,423$ per QALY gained

- ICER (c) vs (a): \$2560 per QALY gained - (b) is dominated by (c), high cost and lower QALYs than (c)

$\$ 50,000$ per QALY gained

- (a) is dominated by (c), high cost and lower QALYs than (c)

- (b) is dominated by (c), high cost and lower QALYs than (c)

$€ 65,000$ per QALY gained

- ICER (d) vs (a): €81,500 per QALY gained

- ICER (d) vs (b): €9111 per QALY gained - ICER (d) vs (c): €5972 per QALY gained

$\begin{array}{ll}\$ 50,000 \text { per } & \text { Genotyping with prasugrel vs (a): } \\ \text { QALY gained } & \$ 35,800 \text { per QALY gained } \\ & \cdot \text { Genotyping with ticagrelor vs (a): } \\ & \$ 30,200 \text { per QALY gained }\end{array}$

$\$ 50,000$ per

- ICER (c) vs (a): \$4200 per QALY gained QALY gained

- (b) is dominated by (c), high cost and lower QALYs than (c)

$\$ 50,000$ per QALY gained

- (a) is dominated by (c), (c) lower cost and more effective than (a)

- (b) is dominated by (c), (c) lower cost and more effective than (b)

AUS\$

50,000 per

QALY gained

- ICER (c) vs (a): \$AUS 6346 per QALY gained

- ICER (b) vs (c): \$AUS 22,821 per QALY gained

\$NZ50000 per QALY gained

- (b) is dominated by (a), high cost and lower QALYs than (a)

- ICER (c) vs (a): \$NZ 24,617 per QALY gained

- (b) is dominated by (c), (c) lower cost 
Table 4 Cost-effectiveness results of included studies (Continued)

\begin{tabular}{|c|c|c|c|c|}
\hline No & $\begin{array}{l}\text { Author, Year of } \\
\text { published }\end{array}$ & $\begin{array}{l}\text { study } \\
\text { setting }\end{array}$ & $\begin{array}{l}\text { Target } \\
\text { populations }\end{array}$ & Intervention vs comparator \\
\hline & & & & Test negative: clopidogrel \\
\hline 9 & $\begin{array}{l}\text { Reese E S et al., } \\
2012 \text { [37] }\end{array}$ & USA & $\begin{array}{l}\mathrm{ACS} \text {, recent } \mathrm{Ml} \\
\text { or stroke } \\
\text { undergoing } \\
\mathrm{PCl}\end{array}$ & $\begin{array}{l}\text { (a) clopidogrel } \\
\text { (b) prasugrel } \\
\text { (c) CYP2C19 testing guided } \\
\text { therapy } \\
\text { Test: positive prasugrel; } \\
\text { Test negative clopidogrel }\end{array}$ \\
\hline
\end{tabular}

Drug: statin, Biomarker: pharmacogenetics test ADR: myopathy, rhabdomyolysis

Mitchel et al., Canada lardiovascular
2017 [39]

Drug: allopurinol, Biomarker: HLAB-5801

\section{ADR: SJS/TEN, DRESS}

1 Cheng $\mathrm{H}$ et al., 2018 [41]

China (Han Hyperuricemia

population) and gout

2 Chong et al., Malaysia gout 2018 [40]

3 Jutkowitz et al., USA 2017 [44]

$4 \mathrm{Ke} \mathrm{CH}$ et al., 2017 Taiwan [43]

5 Plumpton CO UK gout et al., 2017 [42]

\section{(a) statin}

(b) genotyping-guided treatment with statin (only patients experiencing musculoskeletal pain are being tested)

(a) allopurinol $100 \mathrm{mg}$ and 600 mg per day

(b) febuxostat $40 \mathrm{mgand} 80 \mathrm{mg}$ per day

(c) HLA-B5801 testing prior to treatment

Test positive: febuxostat

Test negative: allopurinol

(a) allopurinol starting dose 300 mg, target dose $600 \mathrm{mg}$ per day (current practice)

(b) probenecid target dose $2 \mathrm{~g}$ per day

(c) HLA-B5801 testing prior to treatment

Test positive: probenecid Test negative: allopurinol

(a) allopurinol-febuxostat sequential therapy: allopurinol:target dose $300 \mathrm{mg} /$ day and febuxostat: $80 \mathrm{mg} /$ day, (current practice) (b) HLA-B5801 testing prior to treatment

Test positive: febuxostat

Test negative:allopurinol

gout with chronic kidney disease

(a) benzbromarone $100 \mathrm{mg} /$ day (current practice)

(b) allopurinol target dose 100 $\mathrm{mg} /$ day

(c) febuxostat $80 \mathrm{mg} /$ day

(d) HLA-B5801 testing prior to treatment

Test positive: febuxostat or benzbromarone

Test negative: allopurinol

(a) allopurinol target dose $300 \mathrm{mg}$ per day add prophylactic treatment with colchicine (b) HLA-B5801 testing prior to treatment

Test positive: febuxostat: target dose $80 \mathrm{mg}$ per day

Test negative: allopurinol

6 Dong $D$ et al., Singapore gout 2015 [46] (a) allopurinol starting dose 300

$\mathrm{mg}$, target dose $600 \mathrm{mg}$ per day

(b) allopurinol +safety program
CAN $\quad$ (a) is dominated by (b), high cost and

$\$ 6150$ per QALY lower QALYs than (b)

gained $\quad$ (b) would be cost-effective as long as the test costs less than CAN\$906

N/A

- In all 253 patients

- (c) saved 1,384,040 yuan for allopurinol and febuxostat at the lowest dosages

- (c) saved 2,807,770 yuan for allopurinol and febuxostat at the highest dosages

MYR 39,000 or - (b) is dominated by (a), high cost and $\$ 8695$ per QALY lower QALYs than (a)

gained $\quad$ (c) is dominated by (a), high cost and lower QALYs than (a)
NT

$\$ 800,000$ or US

$\$ 25,600$ per

QALY gainedin

2015

$£ 30,000$ per

QALY gained

- ICER (d) vs (a): $£ 44,954$ in the base case and $£ 38,478$ per QALY in patients with chronic renal insufficiency lower QALYs than (a) current practice) - (c) is dominated by (d), high cost and lower QALYs than (d) current practice) - ICER (d) vs (a): NT\$234,610 per QALY gained in the base-case and NT\$ 230,925 per QALY gained in patients with chronic kidney disease
$\$ 50,000$ per QALY gained
- (b) is dominated by (a), high cost and

- -ICER (b) vs (a): \$79,140 per QALY

- (c) is dominated by (b), high cost and lower QALYs than (b) current practice) 
Table 4 Cost-effectiveness results of included studies (Continued)

\begin{tabular}{|c|c|c|c|c|}
\hline No & $\begin{array}{l}\text { Author, Year of } \\
\text { published }\end{array}$ & $\begin{array}{l}\text { study } \\
\text { setting }\end{array}$ & $\begin{array}{l}\text { Target } \\
\text { populations }\end{array}$ & Intervention vs comparator \\
\hline & & & & $\begin{array}{l}\text { (SP) } \\
\text { (c) HLA-B5801 testing prior to } \\
\text { treatment + SP } \\
\text { (d) HLA-B5801 testing prior to } \\
\text { treatment + SP } \\
\text { Test positive: probenecid target } \\
\text { dose } 2 \text { g per day } \\
\text { Test negative: allopurinol } \\
\text { (e) HLA-B5801 testing prior to } \\
\text { treatment } \\
\text { Test positive: probenecid target } \\
\text { dose } 2 \text { g per day } \\
\text { Test negative: allopurinol } \\
\text { (f) no allopurinol (treatment of } \\
\text { acute flares only) }\end{array}$ \\
\hline 7 & $\begin{array}{l}\text { Park DJ et al., } \\
2015 \text { [45] }\end{array}$ & Korea & $\begin{array}{l}\text { gout with } \\
\text { chronic renal } \\
\text { insufficiency }\end{array}$ & $\begin{array}{l}\text { (a) allopurinol starting dose } 100 \\
\text { mg, target dose } 300 \mathrm{mg} \text { per day } \\
\text { (b) HLA-B5801 testing prior to } \\
\text { treatment } \\
\text { Test positive: febuxostat starting } \\
\text { dose } 40 \text { mg, target dose } 80 \mathrm{mg} \text { ) } \\
\text { per day } \\
\text { Test negative: allopurinol }\end{array}$ \\
\hline 8 & $\begin{array}{l}\text { Saokaew et al., } \\
2014 \text { [47] }\end{array}$ & Thailand & gout & $\begin{array}{l}\text { (a) allopurinol starting dose } 300 \\
\text { mg per day } \\
\text { (b) HLA-B5801 testing prior to } \\
\text { treatment } \\
\text { Test positive: probenecid target } \\
\text { dose } 1 \mathrm{mg} \text { target to } 2 \mathrm{~g} \text { per day } \\
\text { Test negative: allopurinol }\end{array}$ \\
\hline
\end{tabular}

Drug: abacavir, Biomarker: HLA B*57:01 ADR: hypersensitivity

1 Kubaeva et al., Russia HIV 2018 [48]

$\begin{array}{ll}\text { Kapoor R et al., } & \begin{array}{l}3 \text { ethnic } \\ \text { groups in } \\ 2015[49]\end{array}\end{array}$

3 Nieves Calatrava Spain HIV et al., 2010 [51]

4 Kauf $T L$ et al.,

USA 2010 [52]
HIV

(a) abacavir regimen

(b) HLA-B* 5701 testing prior to treatment

Test positive: alternative regimens without abacavir

Test negative: abacavir regimen
(a) abacavir-based ART
(b) tenofovir-based ART
(c) HLA-B:5701 testing prior to treatment

Test positive: tenofovir-based ART

Test negative: abacavir-based ART

N/A

$\begin{array}{ll}\begin{array}{l}\text { Cost } \\ \text { effectiveness }\end{array} & \begin{array}{l}\text { Cost-effectiveness results } \\ \text { threshold }\end{array} \\ \end{array}$

threshold

- ICER (d) vs (b): \$85,630 per QALY

- (e) is dominated by (d), high cost and

lower QALYs than (d) current practice)

- (f) is dominated by (d), high cost and

lower QALYs than (d) current practice)

160,000 THB per · ICER (b) vs (a): 156,937 THB per QALY QALY gained

- Costs of (a) \$1193 and (b) \$1055

(b) is less costly and more effective than (a)

\section{$\$ 50,000$ per $\quad$ - Early stage: ICER (c) vs (a):}

QALY gained Chinese $\$ 415,845$ per QALY gained, Malay $\$ 318,029$ per QALY gained, Indian $\$ 208,231$ per QALY gained - Late stage: ICER (c) vs (a): Chinese $\$ 926,938$ per QALY gained, Malay $\$ 624,297$ per QALY gained, Indian \$284,598 per QALY gained

- HIV who are contraindicated to tenofovir

ICER (b) vs (a): all ethnicity was not costeffective, except for Indian patients with early-stage ICER (c) vs (a): \$44,649 per QALY gained

N/A

- cost per HSR avoided of $€ 630$ (a) abacavir regimen

(b) HLA-B:5701 testing prior to treatment

Test positive: alternative HAART regimen without $A B C$

Test negative: abacavir regimen

(a) short-term: abacavirt

lamivudine+efavirenz

(b) long-term:

tenofovir+emtricitabine+efavirenz
$\$ 50,000$ per QALY gained
- Short-term: (c) is dominated by (a), high cost and lower QALYs than (a) - Long-term: (b) is dominated by (c), high cost and lower QALYs than (c) 
Table 4 Cost-effectiveness results of included studies (Continued)

\begin{tabular}{|c|c|c|c|c|c|c|}
\hline No & $\begin{array}{l}\text { Author, Year of } \\
\text { published }\end{array}$ & $\begin{array}{l}\text { study } \\
\text { setting }\end{array}$ & $\begin{array}{l}\text { Target } \\
\text { populations }\end{array}$ & Intervention vs comparator & $\begin{array}{l}\text { Cost } \\
\text { effectiveness } \\
\text { threshold }\end{array}$ & $\begin{array}{l}\text { Cost-effectiveness results } \\
\text { (ICER/ICUR) }\end{array}$ \\
\hline & & & & $\begin{array}{l}\text { (c) HLA-B:5701 testing prior to } \\
\text { treatment } \\
\text { Test positive: } \\
\text { tenofovir+emtricitabine+efavirenz } \\
\text { Test negative: } \\
\text { abacavir+lamivudine+efavirenz; }\end{array}$ & & \\
\hline 5 & $\begin{array}{l}\text { Wolf et al., } 2010 \\
\text { [50] }\end{array}$ & Germany & HIV & $\begin{array}{l}\text { (a) combination of abacavir+ } \\
\text { lamivudine } \\
\text { (b) HLA-B:5701 testing prior to } \\
\text { treatment } \\
\text { Test positive combination of } \\
\text { tenofovir, emtricitabine } \\
\text { Test negative combination of } \\
\text { abacavir+ lamivudine }\end{array}$ & N/A & $\begin{array}{l}\text { - (b) vs (a): (b) cost-saving } € 44 \text { and } 127 \\
\text { per screened patient from healthcare } \\
\text { payer and societal perspective }\end{array}$ \\
\hline 6 & $\begin{array}{l}\text { Schackman BR } \\
\text { et al., } 2008[53]\end{array}$ & USA & HIV & $\begin{array}{l}\text { (a) abacavir-based regimen } \\
\text { (b) tenofovir-based regimen } \\
\text { (c) HLA-B:5701 testing prior to } \\
\text { treatment } \\
\text { Test positive: tenofovir or AZT- } \\
\text { based regimen } \\
\text { Test negative: abacavir-based } \\
\text { regimen }\end{array}$ & $\begin{array}{l}\$ 50,000 \text { per } \\
\text { QALY gained }\end{array}$ & $\begin{array}{l}\text { - ICER (c) vs (a): } \$ 36,700 \text { per QALY } \\
\text { gained } \\
\text { - (b) is dominated by (a),high cost and } \\
\text { lower QALYs than (a) }\end{array}$ \\
\hline 7 & $\begin{array}{l}\text { Hughes DA et al., } \\
2004 \text { [54] }\end{array}$ & UK & HIV & $\begin{array}{l}\text { (a) Trizivir (AZT/3TC/ABC) } \\
\text { (b) HLA-B:5701 testing prior to } \\
\text { treatment } \\
\text { Test positive: HAART regimen } \\
\text { without abacavir } \\
\text { Test negative: Trizivir (AZT/3TC/ } \\
\text { ABC) } \\
\text { (HAART: highly active } \\
\text { antiretroviral therapy) }\end{array}$ & N/A & $\begin{array}{l}\text { - (a) is dominated by (b), high cost and } \\
\text { lower benefit than (b), except for } \\
\text { Trizivir is substituted with } \\
\text { ritonavir+indinavir+combivir } \\
\text { - ICER (b) vs (a): } € 22,811 \text { per } \\
\text { hypersensitivity reaction avoided }\end{array}$ \\
\hline \multicolumn{7}{|c|}{ Drug: efavirenz, Biomarker: CYP2B6, ADRs: sub- or supratherapeutically dosed } \\
\hline 1 & $\begin{array}{l}\text { Schackman et al., } \\
2015 \text { [55] }\end{array}$ & USA & HIV & $\begin{array}{l}\text { (a) efavirenz } 600 \mathrm{mg}+\text { tenofovir+ } \\
\text { emtricitabine } \\
\text { (b) CYP } 2 \mathrm{~B} 6 \text { testing prior to } \\
\text { treatment } \\
\text { Test positive: decrease dose to } \\
\text { 200,400 mg } \\
\text { Test negative: efavirenz } 600 \mathrm{mg} \\
\text { (c) Universal low dose efavirenz } \\
400 \mathrm{mg}+\text { tenofovir+ emtricitabine }\end{array}$ & $\begin{array}{l}\$<100,000 \text { per } \\
\text { QALY gained }\end{array}$ & $\begin{array}{l}\text { - (a) is dominated by (b), high cost and } \\
\text { lower benefit than (b) }\end{array}$ \\
\hline
\end{tabular}

\section{Drug: azathioprine, Biomarker: TPMT ADR: neutropenia or severe neutropenia} et al., 2014 [56]

2 Priest VLet al, New 2006 [58] autoimmune diseases

inflammatory bowel disease (IBD) (a) azathioprine therapy

(b) TPMT testing prior to treatment

Test positive: alternative treatment Test negative: azathioprine

(a) azathioprine

(b) TPMT testing prior to treatment (Phenotype and genotype)
$£ 20,000$ per

QALY gained

N/A

A

50,000 per

Idiopathic Pulmonary Fibrosis (IPF) (a) conservative therapy which no specific therapy

(b) azathioprine+N-acetylcysteine and steroids (no testing)

(c) TPMT testing prior to
- (b) is dominated by (a) lower cost and lower QALYs than (a)

- ICER (b) vs (a): \$49,245 per QALY *ICER (c) vs (b): \$29,663 per QALY gained 
Table 4 Cost-effectiveness results of included studies (Continued)

\begin{tabular}{|c|c|c|c|c|}
\hline No & $\begin{array}{l}\text { Author, Year of } \\
\text { published }\end{array}$ & $\begin{array}{l}\text { study } \\
\text { setting }\end{array}$ & $\begin{array}{l}\text { Target } \\
\text { populations }\end{array}$ & Intervention vs comparator \\
\hline & & & & $\begin{array}{l}\text { treatment } \\
\text { Test positive: conservative } \\
\text { therapy } \\
\text { Test negative: azathioprine+N- } \\
\text { acetylcysteine and steroid }\end{array}$ \\
\hline 4 & $\begin{array}{l}\text { Dubinsky MC } \\
\text { et al., } 2005 \text { [60] }\end{array}$ & USA & $\begin{array}{l}\text { Crohn's } \\
\text { disease }\end{array}$ & $\begin{array}{l}\text { (a) TPMT testing prior to } \\
\text { treatment } \\
\text { Test positive: metrotrexate } \\
\text { Test negative: azathioprine } \\
\text { (b) metabolite monitoring (MM) } \\
\text { prior to treatment with } \\
\text { azathioprin } \\
\text { (c) TPMT testing +MM prior to } \\
\text { treatment with azathioprine } \\
\text { (d) community care (CC) }\end{array}$ \\
\hline 5 & $\begin{array}{l}\text { Winter J et al., } \\
2004 \text { [61] }\end{array}$ & Scotland & $\mathrm{IBD}$ & $\begin{array}{l}\text { (a) azathioprine } \\
\text { (b) TPMT testing prior to } \\
\text { treatment }\end{array}$ \\
\hline
\end{tabular}

Drug: azathioprine, Biomarker: TPMT ADR: all adverse events
6 Sayani FA et al., Canada 2005 [59]
Crohn's disease and IBD
(a) azathioprine
(b) TPMT testing prior to
treatment

$\mathrm{N} / \mathrm{A}$

$\begin{array}{ll}\text { Cost } & \begin{array}{l}\text { Cost-effectiveness results } \\ \text { effectiveness }\end{array} \\ \text { (ICER/ICUR) }\end{array}$

threshold

$\mathrm{N} / \mathrm{A}$ per patient

\section{Drug: azathioprine, Biomarker: TPMT ADR: severe bone marrow toxicity}

7 Oh KT et al., 2004 Korea [62] rheumatoid arthritis and systemic lupus erythematosus (a) azathioprine

(b) TPMT testing prior to treatment
Drug: azathioprine, Biomarker: TPMT ADR: hematological cytopenias
8 Marra CA et al., Canada 2002 [63] rheumatoid arthritis and systemic lupus erythematosus (a) azathioprine

(b) TPMT testing prior to

treatment
N/A

N/A 8982) per QALY gained

(b) sodium valproate (VPA)

(c) HLA-B*15:02 testing prior to treatment

Test positive: VPA

Test negative: carbamazepine

2 Chen et al., 2016 Hong Kong Epilepsy [65]

3 Rattanavipapong Thailand W et al., 2013

[67]
Epilepsy or neuropathic pain (a) current situation, using antiepileptic drug (pre-policy period)

(b) current situation, using antiepileptic drug (post-policy period)

(c) HLA-B*15:02 testing prior to treatment (the ideal situation) (ideal situation)

Test positive: alternative antiepileptic drug

Test negative: carbamazepine or phenytoin

(d) HLA-B*15:02 testing prior to either carbamazepine or phenytoin (extended situation)

(a) carbamazepine (no HLA-B*15:

02 testing)

(b) HLA-B*15:02 testing prior to
$\$ 50,000$ per
QALY gained
- (d) is dominated by (a), (b),and (c), (d) less costs and faster time to response or sustained response - for a 30 year old: ICER (b) vs (a): $£ 347$
per life-year saved and
- for a 60 year old: ICER (b) vs (a): $£ 817$
per life-year saved $\$ 30,011$ per patient and (b) $\$ 34,887$

- (a) is dominated by (b), (b) lower cost and more effective than (a)

- ICER (b) vs (a): \$85,697 per QALY gained

- ICER (c) vs (a): \$11,090 per QALY gained

- ICER (d) vs (a): \$197,158 per QALY gained

120,000 THB per • ICER (b) vs (a):

QALY gained 222,000 THB per QALY for epilepsy, 130,000 THB per QALY for neuropathic 
Table 4 Cost-effectiveness results of included studies (Continued)

\begin{tabular}{|c|c|c|c|c|c|c|}
\hline No & $\begin{array}{l}\text { Author, Year of } \\
\text { published }\end{array}$ & $\begin{array}{l}\text { study } \\
\text { setting }\end{array}$ & $\begin{array}{l}\text { Target } \\
\text { populations }\end{array}$ & Intervention vs comparator & $\begin{array}{l}\text { Cost } \\
\text { effectiveness } \\
\text { threshold }\end{array}$ & $\begin{array}{l}\text { Cost-effectiveness results } \\
\text { (ICER/ICUR) }\end{array}$ \\
\hline & & & & $\begin{array}{l}\text { treatment } \\
\text { Test positive: } \\
\text { for epilepsy: valproate, for } \\
\text { neuropathic pain: gabapentin; } \\
\text { Test negative: carbamazepine } \\
\text { (c) all prescribed alternative (no } \\
\text { HLA-B*15:02 testing) (epilepsy: } \\
\text { valproate, neuropathic pain: } \\
\text { gabapentin) }\end{array}$ & & $\begin{array}{l}\text { pain } \\
\text { • ICER (c) vs (a): } \\
\text { 32,522,000 per QALY for epilepsy, } \\
\text { (epilepsy), 35,877,000 per QALY for } \\
\text { neuropathic pain }\end{array}$ \\
\hline 4 & $\begin{array}{l}\text { Tiamkao S et al., } \\
2013 \text { [66] }\end{array}$ & Thailand & $\begin{array}{l}\text { Epilepsy or } \\
\text { neuropathic } \\
\text { pain and } \\
\text { neurological } \\
\text { diseases }\end{array}$ & $\begin{array}{l}\text { (a) carbamazepine } \\
\text { (b) HLA-B*15:02 testing prior to } \\
\text { treatment } \\
\text { Test positive: non-specified } \\
\text { Test negative: carbamazepine }\end{array}$ & N/A & $\begin{array}{l}\text { - (b) vs (a): (b) was cost-saving 98,54 } \\
\text { THB per } 100 \text { cases of carbamazepi } \\
\text { users }\end{array}$ \\
\hline 5 & $\begin{array}{l}\text { Dong D et al., } \\
2012 \text { [68] }\end{array}$ & Singapore & Epilepsy & $\begin{array}{l}\text { (a) carbamazepine or phenytoin } \\
\text { (b) HLA-B*15:02 testing prior to } \\
\text { treatment } \\
\text { Test positive: valproate; } \\
\text { Test negative carbamazepine or } \\
\text { phenytoin } \\
\text { (c) valproate (no screening) }\end{array}$ & $\begin{array}{l}\$ 50,000 \text { per } \\
\text { QALY gained }\end{array}$ & $\begin{array}{l}\text { ICER (b) vs (a): } \\
\text { Chinese patients } \$ 37,030 \text { per QALY } \\
\text { gained, Malay } \$ 7930 \text { per QALY gain } \\
\text { and Indians } \$ 136,630 \text { per QALY gair } \\
\cdot \text { (c) is dominated by (b), high cost } \\
\text { lower QALYs than (b) }\end{array}$ \\
\hline \multicolumn{7}{|c|}{ Drug: carbamazepine, Biomarker: HLAA*31:01, ADRs: SJS, TENs, hypersensitivity } \\
\hline 1 & $\begin{array}{l}\text { Plumpton et al., } \\
2015 \text { [69] }\end{array}$ & UK & Epilepsy & $\begin{array}{l}\text { (a) carbamazepine } \\
\text { (b) HLA-B*31:01 testing prior to } \\
\text { treatment } \\
\text { Test positive: lamotrigine; } \\
\text { Test negative: carbamazepine }\end{array}$ & $\begin{array}{l}£ 20,000 \text { per } \\
\text { QALY gained }\end{array}$ & $\begin{array}{l}\text { - ICER (b) vs (a): } £ 12,808 \text { per QALY } \\
\text { gained }\end{array}$ \\
\hline
\end{tabular}

\section{Drug: Fluoropyrimidines, Biomarker: DPYD*2A genotype-guided dosing ADR: toxicity iehematologic, GI}

\begin{tabular}{|c|c|c|c|}
\hline $\begin{array}{l}\text { Deenen MJ et al., } \\
2016 \text { [70] }\end{array}$ & $\begin{array}{l}\text { The } \\
\text { Netherland }\end{array}$ & cance & $\begin{array}{l}\text { (a) fluoropyrimidines-based } \\
\text { therapy } \\
\text { (b) DPYD*2A testing prior to } \\
\text { treatment } \\
\text { Test positive: alternative regimen } \\
\text { Test negative: Fluoropyrimidines- }\end{array}$ \\
\hline
\end{tabular}

\section{Drug: irinotecan, Biomarker: UGT1A1 ADR: severe neutropenia}

1 Pichereau $S$ et al., France 2010 [71] metastatic colorectal cancer

Gold HT et al., USA 2009 [72]

metastatic
colorectal

\begin{abstract}
(a) FOLFIRI regimen (5-
fluorouracil, leucovorin and irinotecan)
\end{abstract}

(b) UGT1A1 testing prior to treatment with FOLFIRI Test positive: FOLFOX regimen (oxaliplatine + 5-FU + folinic acid) Test negative: FOLFIRI cancer

(a) FOLFIRI regimen (5-

fluorouracil, leucovorin and irinotecan)
N/A

(b) UGT1A1 testing prior to treatment with FOLFIRI

Test positive: reduce dose to intermediate dose

Test negative: FOLFIRI

Drug: nortriptyline, Biomarker: CYP2D6 ADRs: sub- or supratherapeutic dose

\footnotetext{
1 Bern EJ et al., 2016 [73]
Netherland major depressive
disorder

The
(a) nortriptyline
(b) CYP2D6 genotyping-guided dosing of nortriptyline
}

N/A

$\$ 100,000$ per QALY gained

$€ 50,000$ per QALY gained
- (b) vs (a): (b) cost-savings of €45 (\$61) per patient
- ICER (b) vs (a): to avoid one febrile neutropenia per 1000 patients treated was $€ 9428$ to $€ 10,901$

- ICER (b) vs (a): was cost-effective if the treatment efficacy of irinotecan in homozygotes after dose reduction had to be $\geq 984 \%$ of full-dose efficacy for genetic testing to remain preferred

\section{Drug: oral contraceptive, Biomarker: factor V Leiden ADR: Thromboembolism}

$\begin{array}{llll}1 \text { Smith KJ, et al., USA } & \begin{array}{l}\text { women who } \\ \text { receiving oral }\end{array} & \text { (a) usual care } \\ 2008 \text { [74] genotyping }\end{array}$


Table 4 Cost-effectiveness results of included studies (Continued)

\begin{tabular}{|c|c|c|c|c|c|c|}
\hline No & $\begin{array}{l}\text { Author, Year of } \\
\text { published }\end{array}$ & $\begin{array}{l}\text { study } \\
\text { setting }\end{array}$ & $\begin{array}{l}\text { Target } \\
\text { populations }\end{array}$ & Intervention vs comparator & $\begin{array}{l}\text { Cost } \\
\text { effectiveness } \\
\text { threshold }\end{array}$ & $\begin{array}{l}\text { Cost-effectiveness results } \\
\text { (ICER/ICUR) }\end{array}$ \\
\hline & & & $\begin{array}{l}\text { contraceptives } \\
\text { (OCPs) }\end{array}$ & $\begin{array}{l}\text { (c) genotyping with OCP } \\
\text { counseling } \\
\text { (d) genotyping with OCP } \\
\text { counseling and AC for high-risk } \\
\text { events } \\
\text { (e) genotyping with OCP } \\
\text { counseling and AC for long-term }\end{array}$ & & $\begin{array}{l}\text { - (a) is dominated by }(\mathrm{d}) \text {, high cost and } \\
\text { lower QALYs than }(\mathrm{d}) \\
\text { - (b) is dominated by }(\mathrm{d}) \text {, high cost and } \\
\text { lower QALYs than (d)) }\end{array}$ \\
\hline
\end{tabular}

AC: anticoagulation, ACS: Acute Coronary Syndrome, CBA: Cost-benefit analysis, CEA: Cost-effectiveness analysis, CMA: Cost-minimization analysis, CUA: Costutility analysis, EE: Economic evaluation, ICER: Incremental Cost-Effectiveness Ratio

OCP: oral contraceptive pill, PCl: Percutaneous Coronary Intervention, PSA: Probabilistic Sensitivity Analysis

QALY: Quality adjusted life-year, N/A: Not Applicable, WTP: Willingness to pay

$B * 57: 01$ testing would be cost-effective $[53,54]$ and costsaving $[48,50]$ to prevent HSR due to abacavir as compared with no testing, while the remaining studies showed that it was not cost-effective [51, 52]. In addition, the study in Singapore [48] suggested that genotyping was not cost-effective for Han Chinese and Malays ethnicity but cost-effective in Indian patients. This was because the frequency of the $H L A-B * 5701$ allele and positive predictive value (PPV) in Indians were higher than in Han Chinese and Malays.

CYP2B6 and efavirenz-induced CNS toxicity The study in US demonstrated that the $C Y P 2 B 6$ genotyping before prescribing efavirenz to prevent central nervous system (CNS) toxicity in HIV patients was cost-saving as compared with no testing due to a lower lifetime cost and a gain in QALYs [55].

\section{Autoimmune diseases}

TPMT and azathioprine-induced severe bone marrow toxicity Azathioprine-induced severe bone marrow toxicity was associated with TPMT in patients with autoimmune diseases, inflammatory bowel disease, idiopathic pulmonary fibrosis (IPF), Crohn's disease, rheumatoid arthritis or systemic lupus erythematous. Eight studies were carried out in UK (1 study) [56], New Zealand (1 study) [58], Scotland (1 study) [61], Korea (1 study) [62], Canada (2 studies) [59, 63], and US (2 studies) [57, 60] from 2002 to 2014. These studies employed CUA with model-based (2 studies), [57, 58] CUA with trial-based (1 study) [56], CEA with model-based (2 studies), [60, 62] CEA with trial-based (1 study) [61], CBA with model-based (1 study) [63], and CMA with a randomized prospective study (1 study) [59]. Five studies showed that testing would be a cost-effective [57, 6062] and cost-saving intervention [63] to prevent severe ADRs regarding azathioprine as compared with no testing. Nevertheless, two studies from UK [56] and New Zeland [58] suggested that genotyping would not be cost-effective due to higher costs and lower QALYs than azathioprine therapy without testing. In Canada, it was discovered that genetic testing was not cost-saving [59].

\section{Epilepsy/neuropathic pain}

$H L A-B * 15: 02$ and carbamazepine-induced SJS/TEN Five economic evaluation studies of $H L A-B * 15: 02$ genotyping to prevent the risk of SJS/TEN in patients prescribed carbamazepine (CBZ) were carried out in Malaysia [64], Hong Kong [65], Thailand (2 studies) [66, 67] and Singapore [68] from 2012 to 2017. Four studies applied CUA with model-based economic evaluation $[64,65,67,68]$, while the other study used CBA with retrospective study [66]. All studies focused on the patients diagnosed with epilepsy. Only the study from Thailand [67] included both patients with epilepsy and neuropathic pain. Moreover, the study from Singapore [68] was performed separately for the major ethnic groups, which were Han Chinese, Malays and Indians. Three studies used valproate $[64,67,68]$, while the rest of the studies used any anti-epileptic drug as an alternative for those patients who tested positive with $H L A$ $B * 15: 02[65,66]$.

The findings from three studies showed that a testing would be cost-effective $[65,68]$ and cost-saving to prevent SJS/TEN in CBZ, as compared with no testing [66]. However, a study in Malaysia indicated that testing would not be cost-effective as a result of ethnicity and an effective alternative drug for those who tested positive [64]. The study in Thailand showed that $H L A-B * 15: 02$ screening would be cost-effective in CBZ-treated patients with neuropathic pain but not for epilepsy because the cost of alternative drugs for epilepsy was approximately two times higher than the cost for neuropathic pain [67].

$H L A-A * 31: 01$ and carbamazepine-induced SJS/TEN and hypersensitivity Notably, $\mathrm{CBZ}$ has been associated with $H L A-A * 31: 01$ and it can lead to severe ADRs, such as SJS/TEN and hypersensitivity. A study in UK was performed using CUA with model-based economic 
evaluation in 2015. The results showed that testing would be cost-effective as the efficacy (e.g., remission rate) of anti-epileptic drugs was the main driver of costeffectiveness results [69]. In addition, this study used lamotrigine as an alternative drug for patients who tested positive rather than valproate, which might be different from other clinical settings.

\section{Cancer}

UGT1A1 and irinotecan-induced severe neutropenia One CUA with model-based study from France [71] and one CEA with model-based study from the US [72] were performed to evaluate the cost-effectiveness of UGT1A1 screening before prescribing irinotecan to prevent severe neutropenia in metastatic colorectal cancer. The results demonstrated that genotyping would be a cost-effective intervention.

$D P Y D$ and fluoropyrimidines-induced severe hematologic and GI toxicity One study was conducted in the Netherlands [70] using CBA with model-based economic evaluation in 2016. The results demonstrated that DPYD testing before prescription of fluoropyrimidines would be cost-saving, as compared with no testing, in preventing severe hematologic and GI toxicity due to fluoropyrimidine.

\section{Major depressive disorder}

CYP2D6 and nortriptyline-induced anticholinergic symptoms The CUA with model-based economic evaluation in the Netherland study showed that CYP2D6 screening for adjusting dose before starting nortriptyline compared to no screening would not be cost-effective since CYP2D6 was not potentially related to the reduction of ADRs and to the increased efficacy of nortriptyline in a major depressive disorder [73].

\section{Hormone replacement therapy}

Factor V Leiden and estrogen combined in oral contraceptives-induced thromboembolism The CUA with model-based study in US was conducted to evaluate the cost-effectiveness of Factor V Leiden testing before a prescription of estrogen-containing oral contraceptives to avoid thromboembolism. The study compared testing before prescribing the drug, testing with oral contraceptive pill $(\mathrm{OCP})$ counselling, testing with $\mathrm{OCP}$ counselling and anticoagulation $(\mathrm{AC})$ with the usual care without testing. The results demonstrated that testing with $\mathrm{OCP}$ counselling and prophylactic AC during high-risk periods in female relatives of FVL carriers was costeffective [74].

\section{Uncertainty analysis results}

Based on the results of uncertainty analysis from the included studies, parameters which could influence the cost-effectiveness results are summarized in terms of therapeutic areas and gene-drug pairs in Table 5. These parameters were classified into three types: (1) epidemiological and disease progression parameters, e.g., probability of ADRs related to drug treatment, allele frequency, PPV or negative predictive value (NPV), and mortality rate of ADRs, (2) clinical effectiveness data, e.g., the efficacy of genetic testing and drugs treatment, and (3) resource use and cost parameters, e.g., costs of genetic testing, alternative drugs and hospitalization.

Our review indicated that cost-effectiveness results were mostly sensitive to the probability of drug inducedADRs, the effectiveness of pharmacogenetic testing to prevent ADRs, the cost of testing, and the cost of alternative drugs in patients who tested positive. For instance, in cardiovascular diseases, the probability of MACE due to clopidogrel and the efficacy of CYP2C9 and VKORC1 testing to avoid bleeding complications of warfarin mostly affected the ICER results in clopidogrel and warfarin users, respectively. Furthermore, for HIV infection, the cost of testing had an impact on the ICER results in both abacavir and efavirenz. However, there was no reported uncertainty analysis from a one-way sensitivity analysis among these studies $[18,20,32,37$, $41,48,59,61,66,74]$.

\section{The transferability of economic evaluation results}

Based on Welte et al's method [15] that assesses the transferability of economic evaluation results across countries, three transferability factors were determined from the economic evaluations for $H L A B * 5801$-allopurinol in gout patients [40-47] as a case study. First, methodological characteristics, e.g., perspective, time horizon, cost categories, and discount rate used, varied across CUA studies. Among six CUA studies, a healthcare payer perspective was the most common, followed by a societal perspective. Nevertheless, lifetime horizon was mostly applied. Cost categories and discount rates used were different. Although three main direct medication costs, e.g., the cost of $H L A B * 5801$ testing, cost of treating ADRs and cost of gout maintenance treatment, were mostly included, the cost of flare management of an acute flare or death was considered in some studies. Based on a societal perspective, direct non-medical cost, e.g., transportation cost and additional food cost for patients and their relatives, and indirect costs, e.g., productivity loss due to illness, were incorporated. Costs and outcomes were discounted at a rate of $3 \%$ or $3.5 \%$.

Secondly, the healthcare system characteristics in a particular practice varied among countries. It was reported that China, Taiwan, Korea, UK and US applied 
Table 5 Number of studies reporting parameters which could influence the cost-effectiveness results

\begin{tabular}{|c|c|c|c|c|c|c|c|c|c|}
\hline \multirow[t]{2}{*}{ Therapeutic area } & \multicolumn{4}{|c|}{$\begin{array}{l}\text { Epidemiological and disease progression } \\
\text { parameters }\end{array}$} & \multicolumn{2}{|c|}{ Clinical effectiveness data } & \multicolumn{3}{|c|}{ Resource use and cost parameters } \\
\hline & $\begin{array}{l}\text { probability } \\
\text { of ADRs }\end{array}$ & $\begin{array}{l}\text { allele } \\
\text { frequency }\end{array}$ & $\begin{array}{l}\text { PPV/ } \\
\text { NPV }\end{array}$ & $\begin{array}{l}\text { mortality } \\
\text { rate of } \\
\text { ADRs }\end{array}$ & $\begin{array}{l}\text { efficacy of } \\
\text { genetic } \\
\text { testing }\end{array}$ & $\begin{array}{l}\text { efficacy of } \\
\text { drugs } \\
\text { treatment }\end{array}$ & $\begin{array}{l}\text { cost of } \\
\text { testing }\end{array}$ & $\begin{array}{l}\text { costs of } \\
\text { alternative } \\
\text { drugs }\end{array}$ & $\begin{array}{l}\text { cost of } \\
\text { hospitalization }\end{array}$ \\
\hline \multicolumn{10}{|c|}{ Cardiovascular disease $(n=24)$} \\
\hline CYP2C19 -Clopidogrel & {$[30,33,34]$} & & & [31] & & [35] & & [36] & [38] \\
\hline $\begin{array}{l}\text { CYP2C9 and VKORC1 } \\
\text {-Warfarin }\end{array}$ & {$[17,21,25]$} & & & & $\begin{array}{l}{[16,22,26,} \\
28,29]\end{array}$ & & {$[23,24]$} & {$[19]$} & \\
\hline $\begin{array}{l}\text { pharmacogenetic testing- } \\
\text { Statin }\end{array}$ & & & & & & [39] & & & \\
\hline \multicolumn{10}{|l|}{ Gout $(n=8)$} \\
\hline HLA-B*58:01 -Allopurinol & {$[43,44,46]$} & [45] & & & & [40] & {$[42]$} & {$[44]$} & {$[40,47]$} \\
\hline \multicolumn{10}{|l|}{ HIV infection $(n=8)$} \\
\hline HLA-B*57:01 -Abacavir & & [50] & & [49] & & & {$[51,52]$} & {$[53,54]$} & \\
\hline CYP2B6 -Efavirenz & & & & & & & {$[55]$} & & \\
\hline \multicolumn{10}{|l|}{ Autoimmune disease $(n=8)$} \\
\hline TPMT - Azathioprine & & [57] & [62] & & [63] & & {$[56,58]$} & {$[60]$} & \\
\hline \multicolumn{10}{|c|}{ Epilepsy/neuropathic pain $(n=6)$} \\
\hline $\begin{array}{l}\text { HLA-B*15:02 or HLA-A*31: } \\
01 \text {-Carbamazepine }\end{array}$ & & & $\begin{array}{l}{[67,} \\
68]\end{array}$ & & & {$[64,69]$} & & {$[65,67]$} & \\
\hline \multicolumn{10}{|l|}{ Cancer $(n=3)$} \\
\hline UGT1A1 -Irinotecan & & & & & [71] & [72] & & & \\
\hline DPYD -Fluoropyrimidine & [70] & & & & & & & & \\
\hline \multicolumn{10}{|c|}{ Major depressive disorder $(n=1)$} \\
\hline CYP2D6 -Nortriptyline & & & & & [73] & [73] & & & \\
\hline \multicolumn{10}{|c|}{ Hormone replacement therapy $(n=1)$} \\
\hline $\begin{array}{l}\text { Factor } \vee \text { Leiden-Estrogen } \\
\text { combined in oral } \\
\text { contraceptives }\end{array}$ & & & & & & & & & \\
\hline
\end{tabular}

ADRs: Adverse Drug Reactions, PPV: Positive Predictive Value, NPV: Negative Predictive Value

febuxostat as an alternative drug in the model based on the recommendations of the American College of Rheumatology that allopurinol and febuxostat were firstline agents for the management of gout [77, 78]. However, febuxostat is not regularly used as an alternative drug in the general clinical practice in Malaysia, Thailand and Singapore. Although the same alternative drug was used, the dosage differed across studies. For instance, allopurinol was used starting at either 100 to $600 \mathrm{mg} /$ day or 100 to $300 \mathrm{mg} /$ day in patients with CKD, febuxostat was used at 40 to $80 \mathrm{mg} /$ day, and probenecid was used at $2 \mathrm{~g} /$ day.

Lastly, in terms of population characteristics, disease prevalence was one of the substantial variation factors that could not be transferred from one country to another. The $H L A-B * 5801$ allele frequency and PPV for SJS/TEN were the key drivers influencing costeffectiveness results. Interestingly, the study in US revealed that genotyping would be cost-effective for Asians and African Americans but not for Caucasians or Hispanics because the $H L A-B * 5801$ frequency was varied substantially across racial or ethnic groups which had an impact on the ICERs [44]. Indeed, the $H L A-B * 5801$ allele frequency ranged from $11.9-18.5 \%$ in Asian studies [40, $41,43,45-47]$ and was higher than in US and Europe [42, 44], which ranged from $0.7-3.8 \%$.

Furthermore, the PPV in the Asians was higher than in American and European populations. This implied that Asians who carried HLA-B*5801 allele would have more chances to develop SJS/TEN as compared with Americans and Europeans. In summary, regarding the differences in three potential transferability factors across countries, the costeffectiveness results would be useful for a context specific setting as they may not be directly transferred from one country to another. 


\section{Discussion}

Our study provided the most updated systematic review on economic evaluation studies of pharmacogenetic testing for prevention of ADRs (59 studies) as compared with two previously published systematic reviews in 2008 (7 studies) and 2016 (47 studies). The majority of included studies were conducted in cardiovascular diseases and mostly found in Europe and US; whereas, only one-third of them were performed in Asian countries. Given the fact that the frequency of each genotyping was different across countries, the cost-effectiveness of pharmacogenetic testing would depend on the ethnicity of patients who were receiving the tests. For instance, $H L A-B * 15: 02$ allele is more frequent among Asians than Caucasians, while $H L A-A * 31: 01$ is rarer in Asians, but more frequent in Caucasians. Therefore, the regular screening before starting carbamazepine therapy for $H L A-B * 15: 02$ in Asians is more useful than $H L A-A * 31$ : 01 in the context of clinical implementation and future economic evaluation study. Alongside this, pharmacogenetic testing can prevent drug-induced severe ADRs on clinical outcomes and reduce economic burden, which are considered significant impacts involving the interest of policy-makers and healthcare professionals [2, 4]. Compared with previous published reviews, we included additional economic evaluation studies of other pharmacogenetic testing, such as CYP2D6-nortriptyline, $C Y P 2 B 6$-efavirenz, DPYD- fluoropyrimidines and UGT1A1- irinotecan, and statins.

Our review suggested that CUA and CEA were the most common methods for performing the economic evaluation of pharmacogenetic testing. This is consistent with the recommendation by $\mathrm{Col} \mathrm{NF}$ et al. [79] and Payne $\mathrm{K}$ et al. [80], denoting that economic evaluation methods, i.e., CUA or CEA could capture all relevant costs and benefits of pharmacogenetics testing [79, 80]. In addition, our review on cost-effectiveness results of the pharmacogenetic testing for prevention of ADRs showed differences in the parameters, methods and outcomes among included studies. Consequently, this raised concerns on the transferability of the cost-effectiveness analysis results from one country to another, which has been increasingly recognized due to healthcare resource constraints [15].

Notably, our systematic review shed light on the critical appraisal of all included studies to evaluate the quality in terms of reporting and the source of evidence used for important model input parameters, which had significant impact on cost-effectiveness results. Based on the quality appraisal on reporting economic evaluations according to the CHEERS checklist [14], most studies complied with the checklist, except for single studybased economic evaluations. The finding highlighted that $78 \%$ of the single study-based economic evaluation studies did not report uncertainty analysis results of the parameters affecting cost-effectiveness results. This may be due to the fact that these reports did not indicate confidence intervals which are necessary measures for performing uncertainty analysis. It should be noted that the advantages of the uncertainty analysis surrounding effects and costs are to provide a correct evaluation of the expected effects and costs, to consider whether existing evidence is sufficient, and to assess the possible consequences of an uncertain decision for decision makers [81]. Therefore, it is worth noting that future studies on the economic evaluation of pharmacogenetic testing with single study-based studies should include uncertainty analysis, since this could significantly lead to the robustness of economic evaluation results. Furthermore, our study revealed that there were studies that failed to report funding (20\%) and authors' disclosure of conflicts of interest (COI) (22\%), possibly leading to biased results when making decisions by clinicians, patients and policy-makers, as the authors or funders might have influenced the research findings. Most studies with the omissions of funding sources (67\%) and COI (85\%) were published between 2002 to 2010, when the reporting of this information had not been mandatory by the journal standards.

In addition, our review highlighted two gaps of knowledge that should be considered for assessing the quality of data sources used for pharmacogenetic testing. First, data sources of clinical effectiveness in several therapeutic areas related to pharmacogenetic testing to prevent drug-induced serious ADRs were very limited, which was consistent with the previous review [11]. Nevertheless, we appraised broader data sources of evidence used not only clinical effectiveness data, but also baseline clinical values, costs as well as resources used and utility data. Our results revealed that there was lack of high-quality evidence, not only estimating the clinical effectiveness of pharmacogenetic testing, but also providing baseline clinical data, according to the hierarchy of evidence developed by Cooper et al. [12]. For example, only 16 studies (27\%) obtained clinical effectiveness data of genetic testing from five major RCTs: the PREDICT-1 trial [82] for $H L A-B * 57: 01$-abacavir, the TARGET trial [83] for TPMT-azathioprine, ARIES trial [84] and the COUMAGEN trial [85] for CYP2C9 and VKORC1-warfarin, the PLATO trial [86, 87] for CYP2C19-clopidogrel. Yet, of all RCTs, only two RCTs supported pharmacogenetic testing to prevent severe ADRs induced by abacavir and azathioprine. Interestingly, we found that eight studies (14\%) obtained clinical effectiveness of testing from the meta-analysis of case-control study with direct comparison, which is not listed in the hierarchy of data sources by Cooper et al. [12]. 
Second, there were very limited baseline clinical data on pharmacogenetic testing. Our review revealed that only five studies (9\%) explicitly analyzed baseline clinical data from reliable databases, including patients from the study setting given that such specific database included patients who developed severe ADRs, which are rare events that might not be commonly available. It should be noted that the quality of sources, especially for clinical effectiveness and baseline clinical data, used to evaluate the economic evaluations of pharmacogenetic testing would be relatively different from that of pharmaceutical interventions. Consequently, this could shed light on a specific ranking system for quality of evidence which is needed for economic evaluation of pharmacogenetic testing to prevent ADRs.

Our study had several strengths. First, pharmacogenetic testing and drug-related ADRs were selected based on the list of currently available clinical guidelines and approved drug labels. Thus, only studies related to treatment options in clinical practice were included to ensure a significant benefit of pharmacogenetic testing and might be useful for clinical decision-making and policy implementation. Second, we added several pharmacogenetic testing from previous studies, such as CYP2D6nortriptyline, CYP2B6-efavirenz, DPYD-fluoropyrimidines, UGT1A1- irinotecan, and pharmacogenetic testing for statins. Third, we appraised the quality of included studies for both the quality of reporting and data sources of evidence used which had a broader component than the previous studies. This review also described in detail the differences in parameters, methods and economic evaluation results of included studies. Furthermore, we demonstrated a case study to evaluate the transferability of the study results across countries according to potential transferability factors to inform that the economic evaluations of pharmacogenetic testing would be useful for a specific setting or might not been transferred to other clinical settings. The above hypotheses have been supported by the clearly established evidence demonstrating that race/ethnicity and geographic region were possible influencers on the prevalence of HLA-B*5801, in which the prevalence of HLA-B*5801 (<1\%) was found to be lower in Caucasians and Hispanics than that in African Americans (3.8\%) and Asians (7.4\%) [1, 14].

It is significant to address some limitations in our study. First, we evaluated the quality of data sources for model input parameters from the only existing published criteria for economic evaluation study developed by Cooper et al. and the quality of reporting using the CHEERS checklist guidelines. However, the ranking of data sources may not be specific to the economic evaluations of pharmacogenetic testing. To the best of our knowledge, there have been existing published guidelines of the International Society for Pharmacoeconomics and Outcomes Research relevant to this topic [88-92], in which we did not apply them to our study. It is recommended that those guidelines could be used as criteria in future studies. Second, some studies did not report uncertainty analysis results, which could affect costeffectiveness results, therefore we could consider only the results of one-way sensitivity analysis obtained from included studies.

\section{Conclusions}

This comprehensive review found fifty-nine economic evaluations of pharmacogenetic testing to avoid druginduced severe ADRs, which mostly focused on therapeutic areas of cardiovascular diseases. CUA and CEA were commonly applied to perform the economic evaluation of pharmacogenetic testing to prevent druginduced ADRs. Based on the quality appraisal on reporting economic evaluations according to the CHEERS checklist guidelines [14], most studies complied with the guidelines, except that uncertainty analysis of single study-based economic evaluations should be reported. The quality of evidence used in clinical effectiveness data and the baseline clinical data were considered to be lowquality according to the hierarchy of evidence proposed by Cooper et al. Therefore, the criteria for assessing the quality of evidence used for economic evaluation of pharmacogenetic testing of ADRs are needed to be further developed. Differences in parameters, methods and outcomes across studies as well as population-level and system-level differences may lead to the difficulty of comparing cost-effectiveness results across countries. Our findings might be useful for developing future and robust cost-effectiveness analyses of pharmacogenetic testing to inform policy-makers on how to allocate resources effectively and implement such testing into clinical practice.

\section{Abbreviations}

ADRs: Adverse drug reactions; CHEERS: Consolidated Health Economic Evaluation Reporting Standards; SJS: Stevens-Johnson syndrome; TEN: Toxic epidermal necrolysis; CPIC: Clinical Pharmacogenetics Implementation Consortium; DPWG: Royal Dutch Association for the Advancement of Pharmacy - Pharmacogenetics Working Group; CPNDS: Canadian Pharmacogenomics Network for Drug Safety; US FDA: United States Food and Drug Administration; EMA: European Medicines Agency; HCSC: US Health Care Service Corporation; PMDA: Pharmaceuticals and Medical Devices Agency; PRISMA: Preferred Reporting Items for Systematic Reviews and Meta-Analysis; CRD: Centre for Reviews and Dissemination; NHS EED: National Health Service Economic Evaluation Database; CEA: Costeffectiveness analysis; CUA: Cost-utility analysis; CBA: Cost-benefit analysis; CMA: Cost-minimization analysis; QALY: Quality adjusted life year; LY: Life year; N/A: Not applicable; RCTs: Randomized controlled trials; CDSR: Cochrane Database of Systematic Reviews; HIV: Human immunodeficiency virus; DRESS: Drug reaction with eosinophilia and systemic symptoms; OCP: Oral contraceptive pill; PPV: Positive predictive value; ICER: Incremental cost-effectiveness ratio 


\section{Supplementary Information}

The online version contains supplementary material available at https://doi. org/10.1186/s12913-021-07025-8.

Additional file $\mathbf{1}$ Table S1. Search strategies A) Search strategies in MEDLINE via PubMed and CRD's NHS Economic Evaluation Database (NHS EED) and B) Search strategies in Scopus. Table S2. General characteristics and results of the included studies. Table S3. The assessment of quality of reporting using CHEERS checklist [14].

\section{Acknowledgments}

We would like to thank Assist. Prof. Dr. Wanvisa Udomsinprasert for her excellent proofreading of this manuscript. We would like to acknowledge the Faculty of Pharmacy, Mahidol University for supporting the research facility.

\section{Authors' contributions}

ST and OR performed systematic reviews, quality assessment, and interpretation as well as drafted and revised the paper. JJ, KB, and OW participated in the analysis and interpretation of data and the drafting and revising of the paper. UC involved in the conception and design, analysis and interpretation of the data, the drafting of the paper and revising it critically for intellectual content. All authors granted the final approval of the version to be published and agreed to be accountable for all aspects of the work

\section{Funding}

This work is a part of a study in the Doctor of Philosophy program in Social, Economic and Administrative Pharmacy (SEAP), Faculty of Pharmacy, Mahidol University, Bangkok. Besides, this study was supported by grants from the International Research Network-the Thailand Research Fund (IRN60W003) and Health Systems Research Institute (HSRI61-080), Thailand. However, the funders had no role in the design, conduct of the study; collection, management, analysis and interpretation of data, and preparation of the manuscript.

\section{Availability of data and materials}

Not Applicable.

\section{Declarations}

\section{Ethics approval and consent to participate}

Not applicable.

\section{Consent for publication}

Not applicable.

\section{Competing interests}

The authors declare that they have no competing interests.

\section{Author details}

'Social, Economic and Administrative Pharmacy (SEAP) Graduate Program, Department of Pharmacy, Faculty of Pharmacy, Mahidol University, Bangkok, Thailand. ${ }^{2}$ Department of Biochemistry, Faculty of Pharmacy, Mahidol University, Bangkok, Thailand. ${ }^{3}$ Health Economics and Health Technology Assessment (HEHTA), Institute of Health and Wellbeing, University of Glasgow, Glasgow, UK. ${ }^{4}$ Social and Administrative Pharmacy Division, Department of Pharmacy, Faculty of Pharmacy, Mahidol University, 447 Sri-Ayuthaya Rd, Payathai, Ratchathewi, Bangkok 10400, Thailand. ${ }^{5}$ Mahidol University Health Technology Assessment (MUHTA) Graduate Program, Mahidol University, Bangkok, Thailand.

Received: 17 December 2020 Accepted: 14 September 2021 Published online: 02 October 2021

\section{References}

1. Lazarou J, Pomeranz BH, Corey PN. Incidence of adverse drug reactions in hospitalized patients: a meta-analysis of prospective studies. JAMA. 1998; 279(15):1200-5. https://doi.org/10.1001/jama.279.15.1200.
2. The European Commission document "Strengthening pharmacovigilance to reduce adverse effects of medicines.: http://europa.eu/rapid/press-release MEMO-08-782 en.htm?locale=en (2008). Accessed.

3. Negrini S, Becquemont L. HLA-associated drug hypersensitivity and the prediction of adverse drug reactions. Pharmacogenomics. 2017;18(15):144157. https://doi.org/10.2217/pgs-2017-0090.

4. Ernst FR, Grizzle AJ. Drug-related morbidity and mortality: updating the cost-of-illness model. J Am Pharm Assoc (Wash). 2001;41(2):192-9. https:// doi.org/10.1016/S1086-5802(16)31229-3.

5. Ingelman-Sundberg M. Pharmacogenetics of cytochrome P450 and its applications in drug therapy: the past, present and future. Trends Pharmacol Sci. 2004;25(4):193-200. https://doi.org/10.1016/j.tips.2004.02.007.

6. Whirl-Carrillo M, McDonagh EM, Hebert JM, Gong L, Sangkuhl K, Thorn CF, et al. Pharmacogenomics knowledge for personalized medicine. Clin Pharmacol Ther. 2012;92(4):414-7. https://doi.org/10.1038/clpt.2012.96.

7. Drug label annotations. https://www.pharmgkb.org/labelAnnotations Accessed 1st August 2020.

8. Hoffmann C. Graf von der Schulenburg JM. The influence of economic evaluation studies on decision making. A European survey. The EUROMET group. Health Policy. 2000;52(3):179-92. https://doi.org/10.1016/s0168-851 0(00)00076-2.

9. Hoffmann C, Stoykova BA, Nixon J, Glanville JM, Misso K, Drummond MF. Do health-care decision makers find economic evaluations useful? The findings of focus group research in UK health authorities. Value Health. 2002:5(2):71-8. https://doi.org/10.1046/j.1524-4733.2002.52109.x.

10. Compagni A, Bartoli S, Buehrlen B, Fattore G, Ibarreta D, de Mesa EG. Avoiding adverse drug reactions by pharmacogenetic testing: a systematic review of the economic evidence in the case of TPMT and AZA-induced side effects. Int J Technol Assess Health Care. 2008;24(3):294-302. https:// doi.org/10.1017/s0266462308080392.

11. Plumpton CO, Roberts D, Pirmohamed M, Hughes DA. A systematic review of economic evaluations of Pharmacogenetic testing for prevention of adverse drug reactions. Pharmacoeconomics. 2016;34(8):771-93. https://doi. org/10.1007/s40273-016-0397-9.

12. Cooper N, Coyle D, Abrams K, Mugford M, Sutton A. Use of evidence in decision models: an appraisal of health technology assessments in the UK since 1997. J Health Serv Res Policy. 2005;10(4):245-50. https://doi.org/10.12 58/135581905774414187.

13. Moher D, Liberati A, Tetzlaff J, Altman DG. Preferred reporting items for systematic reviews and meta-analyses: the PRISMA statement. J Clin Epidemiol. 2009;62(10):1006-12. https://doi.org/10.1016/j.jclinepi.2009.06. 005.

14. Husereau D, Drummond M, Petrou S, Carswell C, Moher D, Greenberg D, et al. Consolidated health economic evaluation reporting standards (CHEERS)-explanation and elaboration: a report of the ISPOR health economic evaluation publication guidelines good reporting practices task force. Value Health. 2013; 16(2):231-50. https://doi.org/10.1016/j.jval.2013.02.002.

15. Welte $R$, Feenstra $T$, Jager $H$, Leidl R. A decision chart for assessing and improving the transferability of economic evaluation results between countries. Pharmacoeconomics. 2004;22(13):857-76. https://doi.org/10.2165/ 00019053-200422130-00004

16. Kim DJ, Kim HS, Oh M, Kim EY, Shin JG. Cost effectiveness of genotypeguided warfarin dosing in patients with mechanical heart valve replacement under the fee-for-service system. Appl Health Econ Health Policy. 2017;15(5):657-67. https://doi.org/10.1007/s40258-017-0317-y.

17. Verhoef TI, Redekop WK, Langenskiold S, Kamali F, Wadelius M, Burnside G, et al. Cost-effectiveness of pharmacogenetic-guided dosing of warfarin in the United Kingdom and Sweden. Pharmacogenomics J. 2016;16(5):478-84. https://doi.org/10.1038/tpj.2016.41.

18. Mitropoulou C, Fragoulakis V, Bozina N, Vozikis A, Supe S, Bozina T, et al. Economic evaluation of pharmacogenomic-guided warfarin treatment for elderly Croatian atrial fibrillation patients with ischemic stroke. Pharmacogenomics. 2015;16(2):137-48. https://doi.org/10.2217/pgs.14.167.

19. You JH. Pharmacogenetic-guided selection of warfarin versus novel oral anticoagulants for stroke prevention in patients with atrial fibrillation: a costeffectiveness analysis. Pharmacogenet Genomics. 2014;24(1):6-14. https:// doi.org/10.1097/fpc.0000000000000014.

20. Pink J, Pirmohamed M, Lane S, Hughes DA. Cost-effectiveness of pharmacogenetics-guided warfarin therapy vs. alternative anticoagulation in atrial fibrillation. Clin Pharmacol Ther. 2014;95(2):199-207. https://doi.org/1 $0.1038 /$ clpt.2013.190 
21. Chong HY, Saokaew S, Dumrongprat K, Permsuwan U, Wu DB, Sritara P, et al. Cost-effectiveness analysis of pharmacogenetic-guided warfarin dosing in Thailand. Thromb Res. 2014;134(6):1278-84. https://doi.org/10.1016/j. thromres.2014.10.006.

22. You JH, Tsui KK, Wong RS, Cheng G. Cost-effectiveness of dabigatran versus genotype-guided management of warfarin therapy for stroke prevention in patients with atrial fibrillation. PLoS One. 2012;7(6):e39640. https://doi.org/1 0.1371/journal.pone.0039640

23. Meckley LM, Gudgeon JM, Anderson JL, Williams MS, Veenstra DL. A policy model to evaluate the benefits, risks and costs of warfarin pharmacogenomic testing. Pharmacoeconomics. 2010;28(1):61-74. https:// doi.org/10.2165/11318240-000000000-00000.

24. You JH, Tsui KK, Wong RS, Cheng G. Potential clinical and economic outcomes of CYP2C9 and VKORC1 genotype-guided dosing in patients starting warfarin therapy. Clin Pharmacol Ther. 2009;86(5):540-7. https://doi. org/10.1038/clpt.2009.104.

25. Patrick AR, Avorn J, Choudhry NK. Cost-effectiveness of genotype-guided warfarin dosing for patients with atrial fibrillation. Circ Cardiovasc Qual Outcomes. 2009;2(5):429-36. https://doi.org/10.1161/circoutcomes.108. 808592

26. Eckman MH, Rosand J, Greenberg SM, Gage BF. Cost-effectiveness of using pharmacogenetic information in warfarin dosing for patients with nonvalvular atrial fibrillation. Ann Intern Med. 2009;150(2):73-83. https://doi. org/10.7326/0003-4819-150-2-200901200-00005.

27. McWilliam A, Lutter $R$, Nardinelli $C$. Healthcare impact of personalized medicine using genetic testing: an exploratory analysis for warfarin. Per Med. 2008:5(3):279-84. https://doi.org/10.2217/17410541.5.3.279.

28. Schalekamp T, Boink GJ, Visser LE, Stricker BH, de Boer A, Klungel OH. CYP2C9 genotyping in acenocoumarol treatment: is it a cost-effective addition to international normalized ratio monitoring? Clin Pharmacol Ther. 2006;79(6):511-20. https://doi.org/10.1016/j.clpt.2006.03.008

29. You JH, Chan FW, Wong RS, Cheng G. The potential clinical and economic outcomes of pharmacogenetics-oriented management of warfarin therapy a decision analysis. Thromb Haemost. 2004;92(3):590-7. https://doi.org/10.11 60/th04-03-0161.

30. Wang Y, Yan BP, Liew D, Lee WWY. Cost-effectiveness of cytochrome P450 $2 \mathrm{C} 19 * 2$ genotype-guided selection of clopidogrel or ticagrelor in Chinese patients with acute coronary syndrome. Pharmacogenomics J. 2018;18(1): 113-20. https://doi.org/10.1038/tpj.2016.94.

31. Jiang M, You JH. CYP2C19 LOF and GOF-guided antiplatelet therapy in patients with acute coronary syndrome: a cost-effectiveness analysis. Cardiovasc Drugs Ther. 2017;31(1):39-49. https://doi.org/10.1007/s10557-01 6-6705-y.

32. Deiman BALM, Tonino PAL, Kouhestani K, Schrover CEM, Scharnhorst $V$, Dekker LRC, et al. Reduced number of cardiovascular events and increased cost-effectiveness by genotype-guided antiplatelet therapy in patients undergoing percutaneous coronary interventions in the Netherlands. Neth Heart J. 2016;24(10):589-99. https://doi.org/10.1007/s12471-016-0873-z.

33. Patel V, Lin FJ, Ojo O, Rao S, Yu S, Zhan L, et al. Cost-utility analysis of genotype-guided antiplatelet therapy in patients with moderate-to- high risk acute coronary syndrome and planned percutaneous coronary intervention. Pharm Pract (Granada). 2014;12(3):438. https://doi.org/10.4321/ S1886-36552014000300007

34. Kazi DS, Garber AM, Shah RU, Dudley RA, Mell MW, Rhee C, et al. Costeffectiveness of genotype-guided and dual antiplatelet therapies in acute coronary syndrome. Ann Intern Med. 2014;160(4):221-32. https://doi.org/10. 7326/m13-1999.

35. Sorich MJ, Horowitz JD, Sorich W, Wiese MD, Pekarsky B, Karnon JD. Costeffectiveness of using CYP2C19 genotype to guide selection of clopidogrel or ticagrelor in Australia. Pharmacogenomics. 2013;14(16):2013-21. https:// doi.org/10.2217/pgs.13.164

36. Lala A, Berger JS, Sharma G, Hochman JS, Scott Braithwaite R, Ladapo JA. Genetic testing in patients with acute coronary syndrome undergoing percutaneous coronary intervention: a cost-effectiveness analysis. J Thromb Haemost. 2013;11(1):81-91. https://doi.org/10.1111/jth.12059.

37. Reese ES, Daniel Mullins C, Beitelshees AL, Onukwugha E. Cost-effectiveness of cytochrome P450 2C19 genotype screening for selection of antiplatelet therapy with clopidogrel or prasugrel. Pharmacotherapy. 2012;32(4):323-32. https://doi.org/10.1002/j.1875-9114.2012.01048.

38. Panattoni L, Brown PM, Te Ao B, Webster M, Gladding P. The cost effectiveness of genetic testing for CYP2C19 variants to guide thienopyridine treatment in patients with acute coronary syndromes: a New Zealand evaluation. Pharmacoeconomics. 2012;30(11):1067-84. https://doi. org/10.2165/11595080-000000000-00000.

39. Mitchell D, Guertin JR, lliza AC, Fanton-Aita F, LeLorier J. Economic evaluation of a pharmacogenomics test for statin-induced myopathy in cardiovascular high-risk patients initiating a statin. Mol Diagn Ther. 2017; 21(1):95-105. https://doi.org/10.1007/s40291-016-0238-8.

40. Chong HY, Lim YH, Prawjaeng J, Tassaneeyakul W, Mohamed Z, Chaiyakunapruk N. Cost-effectiveness analysis of HLA-B*58: 01 genetic testing before initiation of allopurinol therapy to prevent allopurinolinduced Stevens-Johnson syndrome/toxic epidermal necrolysis in a Malaysian population. Pharmacogenet Genomics. 2018;28(2):56-67. https:// doi.org/10.1097/fpc.0000000000000319.

41. Cheng H, Yan D, Zuo X, Liu J, Liu W, Zhang Y. A retrospective investigation of HLA-B*5801 in hyperuricemia patients in a Han population of China. Pharmacogenet Genomics. 2018;28(5):117-24. https://doi.org/10.1097/fpc. 0000000000000334

42. Plumpton CO, Alfirevic A, Pirmohamed M, Hughes DA. Cost effectiveness analysis of HLA-B*58:01 genotyping prior to initiation of allopurinol for gout. Rheumatology (Oxford). 2017;56(10):1729-39. https://doi.org/10.1093/ rheumatology/kex253.

43. Ke CH, Chung WH, Wen YH, Huang YB, Chuang HY, Tain YL, et al. Costeffectiveness analysis for genotyping before allopurinol treatment to prevent severe cutaneous adverse drug reactions. J Rheumatol. 2017;44(6): 835-43. https://doi.org/10.3899/jrheum.151476.

44. Jutkowitz E, Dubreuil M, Lu N, Kuntz KM, Choi HK. The cost-effectiveness of $\mathrm{HLA}-\mathrm{B}^{*} 5801$ screening to guide initial urate-lowering therapy for gout in the United States. Semin Arthritis Rheum. 2017;46(5):594-600. https://doi. org/10.1016/j.semarthrit.2016.10.009.

45. Park DJ, Kang JH, Lee JW, Lee KE, Wen L, Kim TJ, et al. Cost-effectiveness analysis of HLA-B5801 genotyping in the treatment of gout patients with chronic renal insufficiency in Korea. Arthritis Care Res (Hoboken). 2015;67(2): 280-7. https://doi.org/10.1002/acr.22409.

46. Dong D, Tan-Koi WC, Teng GG, Finkelstein E, Sung C. Cost-effectiveness analysis of genotyping for HLA-B*5801 and an enhanced safety program in gout patients starting allopurinol in Singapore. Pharmacogenomics. 2015; 16(16):1781-93. https://doi.org/10.2217/pgs.15.125.

47. Saokaew S, Tassaneeyakul W, Maenthaisong R, Chaiyakunapruk N. Costeffectiveness analysis of HLA-B*5801 testing in preventing allopurinolinduced SJS/TEN in Thai population. PLoS One. 2014;9(4):e94294. https://doi. org/10.1371/journal.pone.0094294.

48. Kubaeva MB, Gushchina JS, Loskutova EE. Cost-effectiveness of HLA-B*5701 prospective genetic screening of hypersensitivity to abacavir. Asian J Pharmaceutical Clin Res. 2018;11(2):281-3. https://doi.org/10.22159/ajpcr.201 8.v11i2.22519.

49. Kapoor R, Martinez-Vega R, Dong D, Tan SY, Leo YS, Lee CC, et al. Reducing hypersensitivity reactions with HLA-B 5701 genotyping before abacavir prescription: clinically useful but is it cost-effective in Singapore? Pharmacogenet Genomics. 2015;25(2):60-72. https://doi.org/10.1097/fpc. 0000000000000107

50. Wolf E, Blankenburg M, Bogner JR, Becker W, Gorriahn D, Mueller MC, et al. Cost impact of prospective HLA-B*5701-screening prior to abacavir/ lamivudine fixed dose combination use in Germany. Eur J Med Res. 2010; 15(4):145-51. https://doi.org/10.1186/2047-783X-15-4-145.

51. Nieves Calatrava D, Calle-Martin Ode L, Iribarren-Loyarte JA, Rivero-Roman A, Garcia-Bujalance L, Perez-Escolano I, et al. Cost-effectiveness analysis of HLA$B^{*} 5701$ typing in the prevention of hypersensitivity to abacavir in HIV+ patients in Spain. Enferm Infecc Microbiol Clin. 2010;28(9):590-5. https://doi. org/10.1016/j.eimc.2009.09.010.

52. Kauf TL, Farkouh RA, Earnshaw SR, Watson ME, Maroudas P, Chambers MG. Economic efficiency of genetic screening to inform the use of abacavir sulfate in the treatment of HIV. Pharmacoeconomics. 2010;28(11):1025-39. https://doi.org/10.2165/11535540-000000000-00000.

53. Schackman BR, Scott CA, Walensky RP, Losina E, Freedberg KA, Sax PE. The cost-effectiveness of HLA-B*5701 genetic screening to guide initial antiretroviral therapy for HIV. AIDS. 2008;22(15):2025-33. https://doi.org/10.1 097/QAD.0b013e3283103ce6.

54. Hughes DA, Vilar FJ, Ward CC, Alfirevic A, Park BK, Pirmohamed M. Costeffectiveness analysis of HLA B*5701 genotyping in preventing abacavir hypersensitivity. Pharmacogenetics. 2004;14(6):335-42. https://doi.org/10.1 097/00008571-200406000-00002 
55. Schackman BR, Haas DW, Park SS, Li XC, Freedberg KA. Cost-effectiveness of CYP2B6 genotyping to optimize efavirenz dosing in HIV clinical practice. Pharmacogenomics. 2015;16(18):2007-18. https://doi.org/10.2217/pgs.15.142.

56. Thompson AJ, Newman WG, Elliott RA, Roberts SA, Tricker K, Payne K. The cost-effectiveness of a pharmacogenetic test: a trial-based evaluation of TPMT genotyping for azathioprine. Value Health. 2014;17(1):22-33. https:// doi.org/10.1016/j.jval.2013.10.007

57. Hagaman J, Kinder BW, Eckman MH. Thiopurine S- methyltransferase [corrected] testing in idiopathic pulmonary fibrosis: a pharmacogenetic cost-effectiveness analysis. Lung. 2010;188(2):125-32. https://doi.org/10.1007/s00408-009-9217-8.

58. Priest VL, Begg EJ, Gardiner SJ, Frampton CM, Gearry RB, Barclay ML, et al. Pharmacoeconomic analyses of azathioprine, methotrexate and prospective pharmacogenetic testing for the management of inflammatory bowel disease. Pharmacoeconomics. 2006;24(8):767-81. https://doi.org/10.2165/ 00019053-200624080-00004.

59. Sayani FA, Prosser C, Bailey RJ, Jacobs P, Fedorak RN. Thiopurine methyltransferase enzyme activity determination before treatment of inflammatory bowel disease with azathioprine: effect on cost and adverse events. Can J Gastroenterol. 2005;19(3): 147-51. https:/doi.org/10.1155/2005/470863.

60. Dubinsky MC, Reyes E, Ofman J, Chiou CF, Wade S, Sandborn WJ. A costeffectiveness analysis of alternative disease management strategies in patients with Crohn's disease treated with azathioprine or 6mercaptopurine. Am J Gastroenterol. 2005;100(10):2239-47. https://doi.org/1 0.1111/j.1572-0241.2005.41900.x.

61. Winter J, Walker A, Shapiro D, Gaffney D, Spooner RJ, Mills PR. Costeffectiveness of thiopurine methyltransferase genotype screening in patients about to commence azathioprine therapy for treatment of inflammatory bowel disease. Aliment Pharmacol Ther. 2004;20(6):593-9. https://doi.org/10.1111/j.1365-2036.2004.02124.x.

62. Oh KT, Anis AH, Bae SC. Pharmacoeconomic analysis of thiopurine methyltransferase polymorphism screening by polymerase chain reaction for treatment with azathioprine in Korea. Rheumatology. 2004;43(2):156-63. https://doi.org/10.1093/rheumatology/keh001.

63. Marra CA, Esdaile JM, Anis AH. Practical pharmacogenetics: the cost effectiveness of screening for thiopurine s-methyltransferase polymorphisms in patients with rheumatological conditions treated with azathioprine. J Rheumatol. 2002;29(12):2507-12.

64. Chong HY, Mohamed Z, Tan LL, Wu DBC, Shabaruddin FH, Dahlui M, et al. Is universal HLA-B*15:02 screening a cost-effective option in an ethnically diverse population? A case study of Malaysia. Br J Dermatol. 2017;177(4): 1102-12. https://doi.org/10.1111/bjd.15498.

65. Chen Z, Liew D, Kwan P. Real-world cost-effectiveness of pharmacogenetic screening for epilepsy treatment. Neurology. 2016;86(12):1086-94. https:// doi.org/10.1212/wnl.0000000000002484.

66. Tiamkao S, Jitpimolmard J, Sawanyawisuth K, Jitpimolmard S. Cost minimization of HLA-B*1502 screening before prescribing carbamazepine in Thailand. Int J Clin Pharm. 2013;35(4):608-12. https:/doi.org/10.1007/s11096-013-9777-9.

67. Rattanavipapong W, Koopitakkajorn T, Praditsitthikorn N, Mahasirimongkol S, Teerawattananon Y. Economic evaluation of HLA-B*15:02 screening for carbamazepine-induced severe adverse drug reactions in Thailand. Epilepsia. 2013;54(9):1628-38. https://doi.org/10.1111/epi.12325.

68. Dong D, Sung C, Finkelstein EA. Cost-effectiveness of HLA-B*1502 genotyping in adult patients with newly diagnosed epilepsy in Singapore. Neurology. 2012;79(12):1259-67. https://doi.org/10.1212/WNL.0b013e31826aac73.

69. Plumpton CO, Yip VL, Alfirevic A, Marson AG, Pirmohamed M, Hughes DA. Costeffectiveness of screening for HLA-A*31:01 prior to initiation of carbamazepine in epilepsy. Epilepsia. 2015;56(4):556-63. https://doi.org/10.1111/epi.12937.

70. Deenen MJ, Meulendijks D, Cats A, Sechterberger MK, Severens JL, Boot H, et al. Upfront genotyping of DPYD*2A to individualize fluoropyrimidine therapy: a safety and cost analysis. J Clin Oncol. 2016;34(3):227-34. https:// doi.org/10.1200/JCO.2015.63.1325.

71. Pichereau S, Le Louarn A, Lecomte T, Blasco H, Le Guellec C, Bourgoin H. Cost-effectiveness of UGT1A1*28 genotyping in preventing severe neutropenia following FOLFIRI therapy in colorectal cancer. J Pharm Pharm Sci. 2010;13(4):615-25. https://doi.org/10.18433/j3wk5s.

72. Gold HT, Hall MJ, Blinder V, Schackman BR. Cost effectiveness of pharmacogenetic testing for uridine diphosphate glucuronosyltransferase 1A1 before irinotecan administration for metastatic colorectal cancer. Cancer. 2009;115(17):3858-67. https://doi.org/10.1002/cncr.24428.

73. Berm EJ, Gout-Zwart JJ, Luttjeboer J, Wilffert B, Postma MJ. A model based cost-effectiveness analysis of routine genotyping for CYP2D6 among older, depressed inpatients starting nortriptyline pharmacotherapy. PLoS One. 2016;11(12):e0169065. https://doi.org/10.1371/journal.pone.0169065.

74. Smith KJ, Monsef BS, Ragni MV. Should female relatives of factor V Leiden carriers be screened prior to oral contraceptive use? A cost-effectiveness analysis. Thromb Haemost. 2008;100(3):447-52. https:/doi.org/10.1160/TH08-03-0149.

75. The Clinical Pharmacogenetics Implementation Consortium (CPIC). https:// cpicpgx.org Accessed 16 April 2019.

76. Table of Pharmacogenetic Associations. https://www.fda.gov/medicaldevices/precision-medicine/table-pharmacogenetic-associations (2020). Accessed 28 July 2020.

77. Khanna D, Fitzgerald JD, Khanna PP, Bae S, Singh MK, Neogi T, et al. 2012 American College of Rheumatology guidelines for management of gout. Part 1: systematic nonpharmacologic and pharmacologic therapeutic approaches to hyperuricemia. Arthritis care \& research. 2012;64(10):1431-46. https:/doi.org/10.1002/acr.21772.

78. Khanna D, Khanna PP, Fitzgerald JD, Singh MK, Bae S, Neogi T, et al. 2012 American College of Rheumatology guidelines for management of gout. Part 2: therapy and antiinflammatory prophylaxis of acute gouty arthritis. Arthritis care \& research. 2012;64(10):1447-61. https://doi.org/10.1002/acr.21773.

79. Col NF. The use of gene tests to detect hereditary predisposition to chronic disease: is cost-effectiveness analysis relevant? Med Decis Mak. 2003;23(5): 441-8. https://doi.org/10.1177/0272989x03254456.

80. Payne K, Shabaruddin FH. Cost-effectiveness analysis in pharmacogenomics. Pharmacogenomics. 2010;11(5):643-6. https://doi.org/10.2217/pgs.10.45.

81. Claxton K. Exploring uncertainty in cost-effectiveness analysis. Pharmacoeconomics. 2008;26(9):781-98. https://doi.org/10.2165/00019053-200826090-00008.

82. Mallal S, Phillips E, Carosi G, Molina JM, Workman C, Tomazic J, et al. HLAB*5701 screening for hypersensitivity to abacavir. N Engl J Med. 2008;358(6): 568-79. https://doi.org/10.1056/NEJMoa0706135.

83. Newman WG, Payne K, Tricker K, Roberts SA, Fargher E, Pushpakom S, et al. A pragmatic randomized controlled trial of thiopurine methyltransferase genotyping prior to azathioprine treatment: the TARGET study. Pharmacogenomics. 2011;12(6):815-26. https://doi.org/10.2217/pgs.11.32.

84. Young B, Squires K, Patel P, Dejesus E, Bellos N, Berger D, et al. First large, multicenter, open-label study utilizing HLA-B*5701 screening for abacavir hypersensitivity in North America. AIDS. 2008;22(13):1673-5. https://doi. org/10.1097/QAD.0b013e32830719aa.

85. Anderson JL, Horne BD, Stevens SM, Grove AS, Barton S, Nicholas ZP, et al. Randomized trial of genotype-guided versus standard warfarin dosing in patients initiating oral anticoagulation. Circulation. 2007;116(22):2563-70. https://doi.org/10.1161/circulationaha.107.737312.

86. Hulot JS, Collet JP, Montalescot G. Genetic substudy of the PLATO trial. Lancet. 2011; 377(9766):637, author reply -8. doi: https:/doi.org/10.1016/s0140-6736(11)60227-4.

87. Wallentin L, James S, Storey RF, Armstrong M, Barratt BJ, Horrow J, et al. Effect of CYP2C19 and ABCB1 single nucleotide polymorphisms on outcomes of treatment with ticagrelor versus clopidogrel for acute coronary syndromes: a genetic substudy of the PLATO trial. Lancet. 2010;376(9749): 1320-8. https://doi.org/10.1016/s0140-6736(10)61274-3.

88. Brazier J, Ara R, Azzabi I, Busschbach J, Chevrou-Séverac H, Crawford B, et al. Identification, review, and use of health state Utilities in Cost-Effectiveness Models: an ISPOR good practices for outcomes research task force report Value Health. 2019;22(3):267-75. https://doi.org/10.1016/j.jval.2019.01.004.

89. Drummond M, Barbieri M, Cook J, Glick HA, Lis J, Malik F, et al. Transferability of economic evaluations across jurisdictions: ISPOR good research practices task force report. Value Health. 2009;12(4):409-18. https:// doi.org/10.1111/j.1524-4733.2008.00489.x.

90. Garrison LP Jr, Neumann PJ, Erickson P, Marshall D, Mullins CD. Using real-world data for coverage and payment decisions: the ISPOR real-world data task force report. Value Health. 2007;10(5):326-35. https://doi.org/10.1111/j.1524-4733.2007.00186.x

91. Ramsey S, Willke R, Briggs A, Brown R, Buxton M, Chawla A, et al. Good research practices for cost-effectiveness analysis alongside clinical trials: the ISPOR RCT-CEA task force report. Value Health. 2005;8(5):521-33. https://doi. org/10.1111/j.1524-4733.2005.00045.x.

92. Weinstein MC, O'Brien B, Hornberger J, Jackson J, Johannesson M, McCabe $C$, et al. Principles of good practice for decision analytic modeling in healthcare evaluation: report of the ISPOR task force on good research practices-modeling studies. Value Health. 2003;6(1):9-17. https://doi.org/10.1046/j.1 524-4733.2003.00234.x.

\section{Publisher's Note}

Springer Nature remains neutral with regard to jurisdictional claims in published maps and institutional affiliations. 